\title{
Einführung in die Diagnostik humanpathogener Pilze - Teil 2: Identifizierung von Dermatophyten
}

\author{
Introduction to the Diagnosis of Human Pathogenic Fungi - \\ Part 2: Identification of Dermatophytes
}

Autoren

Institut
A.-M. Ksoll, B. Sorhage

Abteilung Dermatologie, Venerologie und Allergologie, Bundeswehrzentralkrankenhaus Koblenz
Bibliografie

DOI http://dx.doi.org/

$10.1055 / \mathrm{s}-0030-1257036$

Akt Dermatol 2011; 37: 391-409

(c) Georg Thieme Verlag KG

Stuttgart · New York

ISSN 0340-2541

Korrespondenzadressen

Anne-Marie Ksoll

Biomed. Fachanalytikerin und Fachlehrerin (LGW-Univ.)

\section{Dr. med. Bernhard Sorhage}

Abteilung Dermatologie, Venerologie und Allergologie Bundeswehrzentralkrankenhaus Koblenz

Akademisches Lehrkrankenhaus der Johannes Gutenberg Universität Mainz Rübernacher Straße 170 56072 Koblenz

\section{Zusammenfassung \\ $\nabla$}

Die Dermatophyten sind keratinophile Pilze und verursachen nur Mykosen der Haut, der Haare und der Nägel. In unverhornte Schichten dringen sie meist nicht ein.

Nach dem Aussehen der asexuellen Fruchtformen bzw. Nebenfruchtformen, den Makroconidien, unterscheidet man die Gattungen Trichophyton, Epidermophyton und Microsporum. Bei Trichophyton und Epidermophyton sind sie glattwandig, bei Microsporum rauwandig. Trichophyton hat walzenförmige, Epidermophyton keulenförmige und Microsporum spindelförmige Makroconidien.

Die Makro- und Mikroconidien sowie eine Reihe anderer charakteristischer Mikrostrukturen werden in der imperfekten (asexuellen) Phase gebildet. Bei vielen Dermatophyten sind außerdem perfekte (ascogene bzw. sexuelle) Fruchtformen, die die Einordnung dieser Hautpilze in das Ord-

\section{Einleitung}

\section{$\nabla$}

Bisher sind etwa 40 Dermatophytenarten bekannt. Charakteristische Mikrostrukturen dieser Hautpilze sind die Makro- und Mikroconidien, ferner Sonderformen des Myzels (Spiral-, Tennisschläger-, Kamm- und Bambushyphen, „Geweihund Kronleuchterformen") sowie Chlamydosporen. Entsprechend der Form der Makroconidien unterscheidet man 3 Dermatophytengattungen:

1. Gattung Trichophyton

Makroconidien glatt- und überwiegend dünnwandig, 3- bis 10-zellig, von zylindrischer, länglicher, manchmal gedrungener, gewulsteter oder unregelmäßiger Form.

Mikroconidien entlang den Hyphen (Akladiumform) oder in Trauben (Botrytisform), rundlich bis keulenförmig, meist sehr zahlreich. nungssystem ermöglichen, nachgewiesen worden. Dermatophyten, die keine sexuellen Fruchtformen bilden oder deren Fruchtformen nicht bekannt sind, werden in einem künstlichen System der „Fungi imperfecti“ (Deuteromycetes) zusammengefasst.

Man unterscheidet geophile Dermatophyten, die als Saprophyten vor allem im Erdboden vorkommen, zoophile Dermatophyten, die bevorzugt tierische Warmblüter befallen, und anthropophile Dermatophyten, die optimal an den Menschen angepasst sind.

Die Pilzerkrankungen durch Dermatophyten, die sogenannten Dermatophytosen, sind - zumindest in unseren westlichen Industrienationen - in deutlichem Zunehmen begriffen und stellen an den Arzt und seine Mitarbeiter besondere Anforderungen, denn eine erfolgreiche antimykotische Therapie setzt immer eine zuvor exakt durchgeführte Pilzdiagnostik voraus.

2. Gattung Epidermophyton

Makroconidien glatt- und dünnwandig, 2- bis 4-zellig, keulenförmig, einzeln entlang den Hyphen angeordnet oder in Büscheln stehend. Es werden keine Mikroconidien gebildet.

3. Gattung Microsporum

Makroconidien zumeist rau- und dickwandig, 4- bis 10-zellig (bei Microsporum nanum jedoch nur 1- bis 3-zellig), spindelförmig oder ellipsoid. Mikroconidien entlang den Hyphen, gelegentlich auch in Traubenform, oval, birnen- oder keulenförmig.

Die Dermatophyten sind durch das gemeinsame Merkmal der Keratinophilie gekennzeichnet und verursachen nur Pilzerkrankungen der Haut, der Haare und der Nägel, die als Dermatophytosen bezeichnet werden.

Der Lebenszyklus einer Reihe von Dermatophyten läuft in 2 verschiedenen Phasen ab. Man unterscheidet eine imperfekte (unvollkommene, 
vegetative bzw. conidiale) Form und eine perfekte (vollkommene bzw. ascogene) Form mit sexueller Fortpflanzung, die für die Einordnung dieser Pilze in das Ordnungssystem ausschlaggebend ist. Nach ihrer ökologischen Herkunft werden anthropophile, zoophile und geophile Dermatophyten unterschieden.

\section{Gattung Trichophyton \\ $\nabla$}

In dieser Gattung sind 22 Arten, darunter die in Mitteleuropa medizinisch weitaus wichtigsten Dermatophyten, Trichophyton rubrum, Trichophyton mentagrophytes und Trichophyton interdigitale zusammengefasst.

\section{Trichophyton ajelloi}

Synonym: Keratinomyces ajelloi

Trichophyton ajelloi ist ein geophiler Dermatophyt von sehr geringer Pathogenität.

Makromorphologie: Schnell wachsende, gelbliche, gelbbraune, orangebraune oder braune, flache Kolonien mit samtiger bis staubig-gipsiger Oberfläche $(\bullet$ Abb. 1 und $\bullet$ Abb.2). Die Kulturunterseite ist rotviolett.

Mikromorphologie: Mikroskopisch charakteristisch für diese Pilzart sind zahlreiche, relativ lange, dickwandige, zylindrischspindelförmige, überwiegend 5- bis 10-zellige Makroconidien (๑ Abb.3).

Mikroconidien sind sehr selten, rundlich bis birnenförmig gestreckt und in Akladiumform angeordnet.

\section{Trichophyton equinum ${ }^{1}$}

\section{Synonym: Ctenomyces equinus}

Trichophyton equinum ist ein zoophiler Dermatophyt, der gelegentlich bei Pferden im Kopf- und Halsbereich eine Pilzinfektion verursacht. Die Infektionskette Tier - Mensch wird nicht oft verifiziert, wie man eigentlich annehmen möchte. Infektionen bei Reitern, Pferdehaltern usw. sind selten [1].

Makromorphologie: Schnell wachsende, weißlich-cremefarbene, flache Kolonien mit samtig-flaumiger, glatter oder zentral gefurchter, im Randbereich meist gelblich pigmentierter Oberfläche ( Abb.4 und $\bullet$ Abb.5). Die Kulturunterseite variiert von gelb über ockergelb und kupferbraun bis dunkelbraun.

Mikromorphologie: Makroconidien sind sehr selten, dünnwandig, zylindrisch-keulenförmig, überwiegend 3- bis 4-zellig und kaum breiter als Hyphenbruchstücke ( $\bullet$ Abb.6).

Mikroconidien sind reichlich vorhanden, rundlich, oval oder birnenförmig und lateral an den Hyphen inseriert. Terminale und interkalare Chlamydosporen werden gelegentlich in älteren Kulturen gebildet.

\section{Trichophyton erinacei}

Synonym: Trichophyton mentagrophytes var. erinacei

Trichophyton erinacei ist ein zoophiler, bei Stacheltieren vorkommender, vor allem auf den Igel spezialisierter Dermatophyt.
Makromorphologie: Schnell wachsende, weiße, flache Kolonien mit puderig-körniger bis gipsiger Oberfläche. Der Kolonienrand ist von sehr feinen, strahlenförmig auslaufenden peripheren Hyphenbündeln umgeben, die oft schwach gelblich pigmentiert sind ( Abb.7). Die Kulturunterseite ist brillantgelb ( $\bullet$ Abb. 8).

Mikromorphologie: Makroconidien sind je nach Stamm in unterschiedlicher Anzahl vorhanden, dünnwandig, zylinderförmig, an den Polen etwas zugespitzt und 2- bis 6-zellig ( $\bullet$ Abb.9). Mikroconidien sind sehr zahlreich, oval bis piriform, lateral an den Hyphen oder in Traubenform angeordnet.

Spiralhyphen werden gelegentlich in wenig lockeren Windungen gebildet ( Abb.10).

\section{Trichophyton gallinae}

Synonym: Achorion gallinae

Trichophyton gallinae ist ein zoophiler Dermatophyt und Erreger des sogenannten „Hühnerfavus“.

Makromorphologie: Mäßig schnell wachsende, weiße bis schwach rosa pigmentierte Kolonien mit feinflaumiger, glatter oder unregelmäßig gefurchter Oberfläche ( Abb.11 und - Abb.12). Der Dermatophyt bildet auf der Kulturunterseite ein erdbeerrotes Pigment, das auch in den Nährboden diffundiert.

Mikromorphologie: Makroconidien sind selten, dünnwandig, zylinderförmig, mit etwas spitz zulaufenden Enden, mitunter leicht gebogen; die Zahl der Kammern schwankt zwischen 3 und 7 ( Abb. 13).

Mikroconidien sind ebenfalls selten, oval bis piriform und lateral an den Hyphen angeordnet.

Chlamydosporen werden oft gebildet und sind je nach Stamm und Alter der Pilzkultur in unterschiedlicher Anzahl vorhanden.

\section{Trichophyton interdigitale}

Bekanntestes Synonym: Trichophyton mentagrophytes var. interdigitale

Dieser anthropophile Dermatophyt befällt in erster Linie die Haut der Hand- und Fußflächen, insbesondere der Zehenzwischenräume. Ausgeprägt ist die Tendenz zum chronischen Befall, der auch zum Übergreifen auf die Nagelsubstanz führen kann [1].

Makromorphologie: Schnell wachsende, weiße Kolonien mit samtig-flaumiger oder watteähnlicher, flockiger, oft zentral leicht eingesunkener Oberfläche ( $\bullet$ Abb.14). Die Kulturunterseite ist farblos, gelegentlich auch hellbraun bis rotbraun pigmentiert. Die Kulturen neigen zur Pleomorphie und bilden nur wenig charakteristische Mikrostrukturen.

Mikromorphologie: Makroconidien sind selten, dünnwandig, zylindrisch-keulenförmig und 2- bis 4-fach septiert ( $\bullet$ Abb.15). Mikroconidien sind vereinzelt bis reichlich vorhanden, rundlich, oval oder birnenförmig, überwiegend lateral an den Hyphen angeordnet, seltener traubenartig in Haufen vorkommend. Spiralhyphen werden nur vereinzelt, vor allem in älteren Kulturen gebildet $($ Abb. 16).

\footnotetext{
${ }^{1}$ Trichophyton equinum wurde mit Trichophyton tonsurans zusammengelegt.
} 


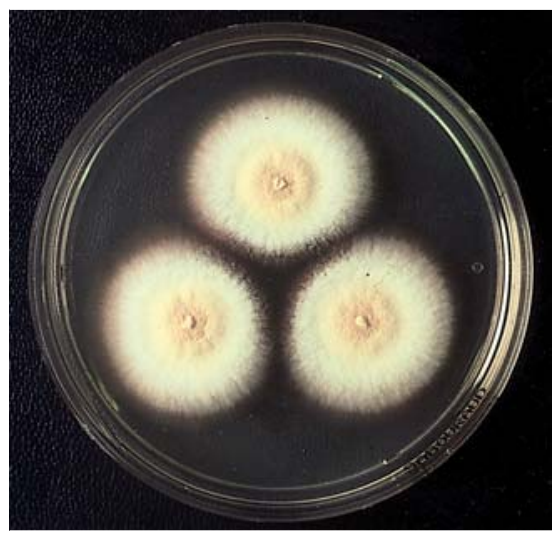

Abb. 1 Trichophyton ajelloi auf SabouraudGlukose-Agar (2\%).

15 Tage alte Drillingskultur mit flacher, puderig-körniger Oberfläche.

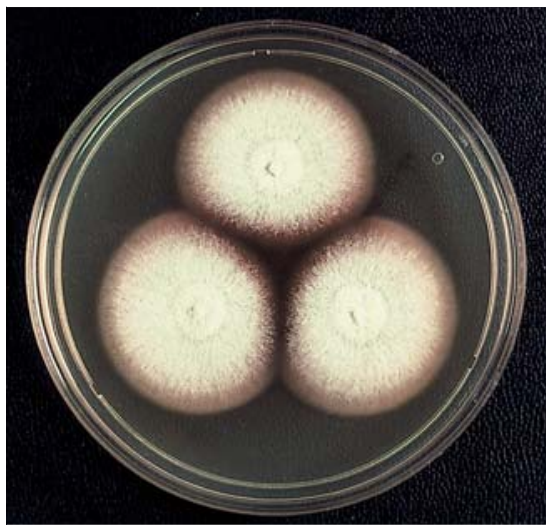

Abb.2 Trichophyton ajelloi auf Mycosel-Agar. 18 Tage alte Drillingskultur mit flacher, feinkörniger Oberfläche und schmalem, hellbraun pigmentiertem Randsaum.

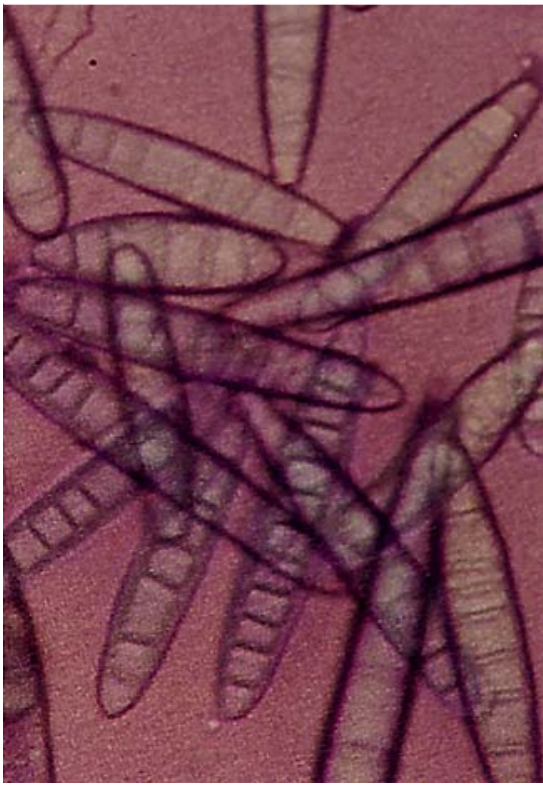

Abb.3 Makroconidien von Trichophyton ajelloi (stark vergrößert).

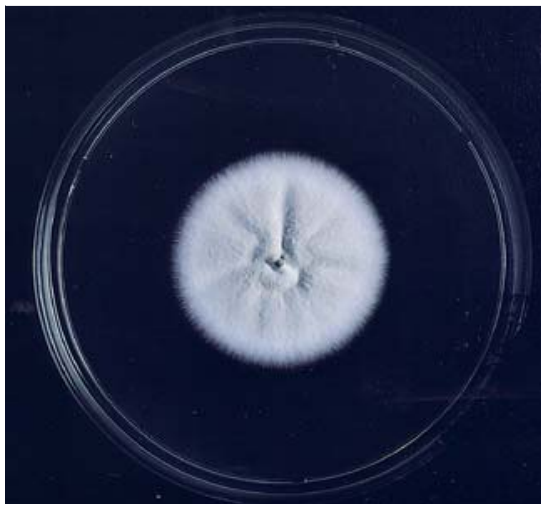

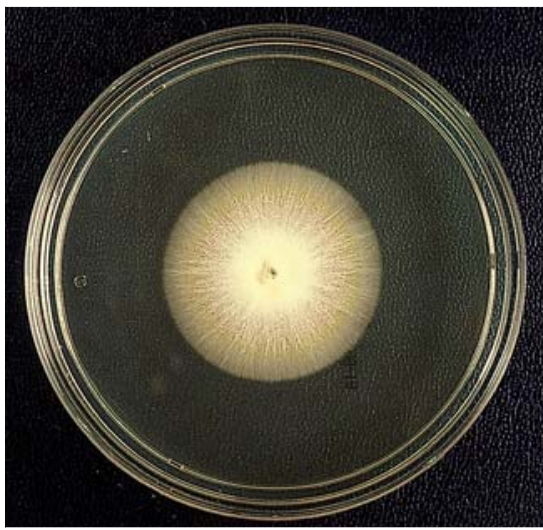

Abb.5 Trichophyton equinum auf MycoselAgar. 21 Tage alte, flache Monokultur mit gelblichem, kurzflaumigem Luftmyzel.

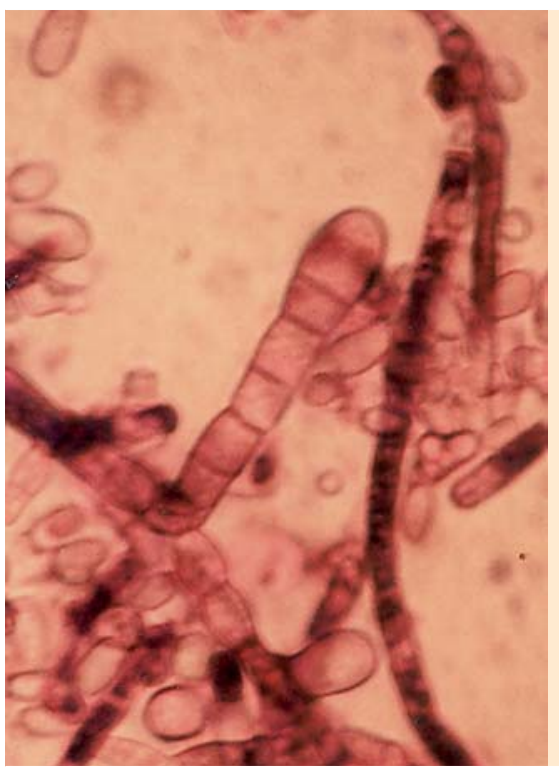

Abb.6 Makroconidie von Trichophyton equinum (stark vergrößert).

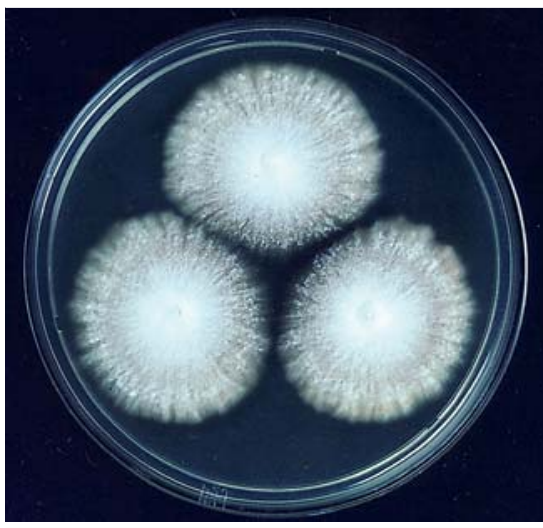

Abb.7 Trichophyton erinacei auf SabouraudGlukose-Agar (2\%).

15 Tage alte, weißlich cremefarbene Drillingskultur mit fein- bis grobkörniger Oberfläche.

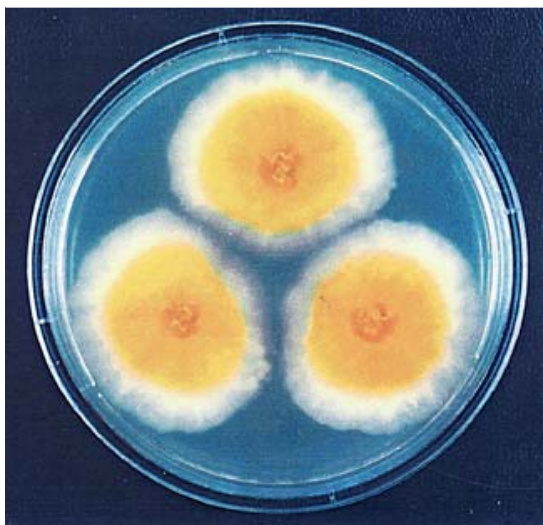

Abb. 8 Brillantgelb pigmentierte Kulturunterseite von Trichophyton erinacei. 

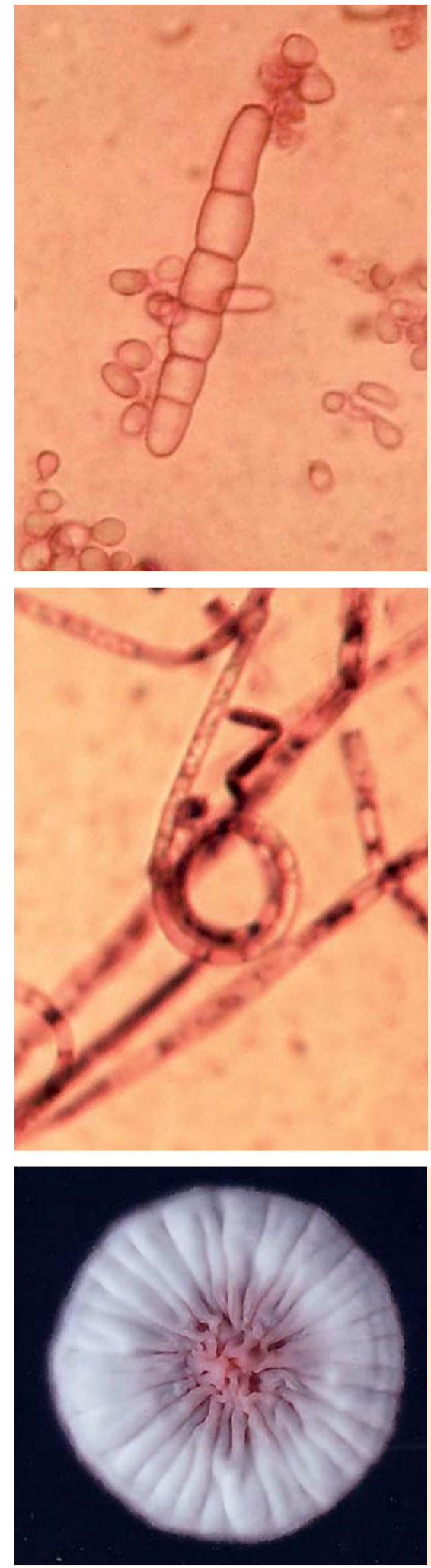

Abb.9 Makroconidie von Trichophyton erinacei (stark vergrößert).

Abb.10 Spiralhyphe von Trichophyton erinacei (stark vergrößert).

Abb.11 Trichophyton gallinae auf KimmigAgar. 25 Tage alte, weiße Monokultur mit samtigem, zentral rosa pigmentiertem, radiär gefurchtem Myzelgeflecht (stark vergrößert).

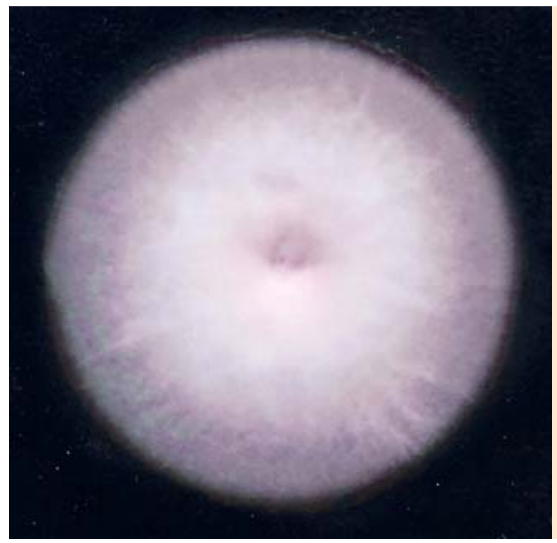

Abb.12 Trichophyton gallinae auf MycoselAgar. 28 Tage alte, flache, hellrosa pigmentierte Monokultur mit kurzflaumigem Luftmyzel (stark vergrößert).

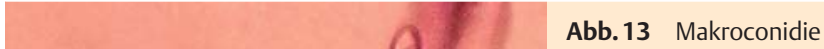

von Trichophyton gallinae (stark vergrößert).
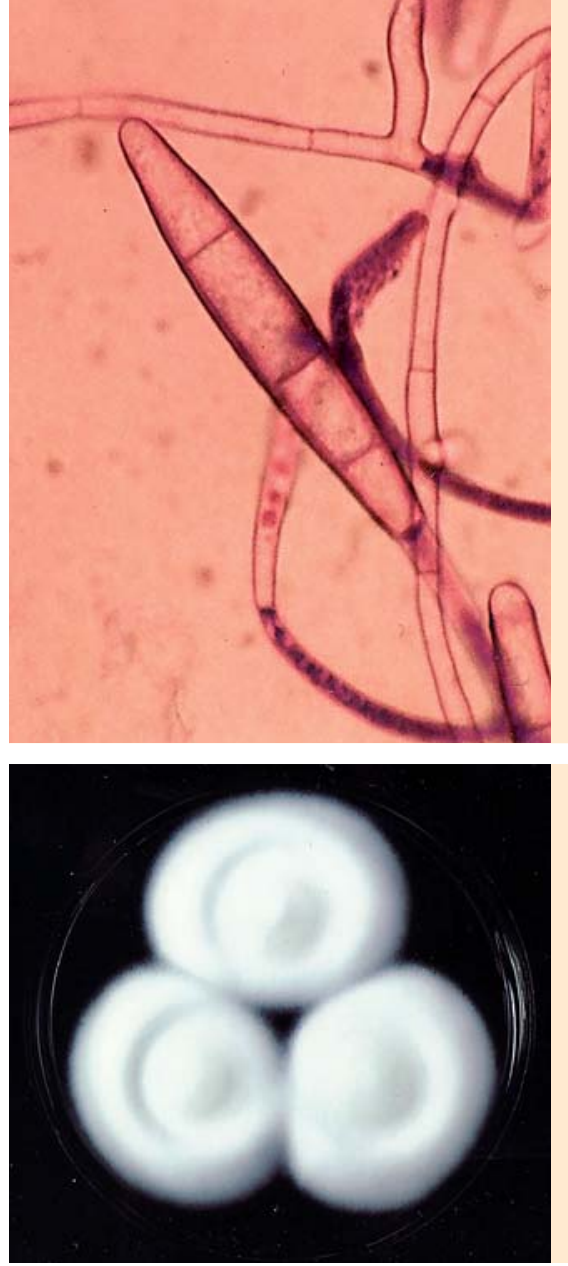

Abb.14 Trichophyton interdigitale auf Sabouraud-Glukose-Agar (2\%). 17 Tage alte, weiße Drillingskultur mit samtig-flaumiger Oberfläche. 


\section{Trichophyton megninii ${ }^{2}$}

Synonym: Trichophyton roseum, Trichophyton rosaceum

Trichophyton megninii ist ein vor allem in Portugal und auf der Insel Sardinien vorkommender, anthropophiler Dermatophyt, der vornehmlich im Bereich des Capillitiums und des Bartes Pilzinfektionen beim Menschen verursacht.

Makromorphologie: Langsam wachsende, anfänglich weiße, später jedoch rosa, rot bis rotbraun pigmentierte Kolonien mit samtig-flaumigem, vielfältig gewundenem oder unregelmäßig radiär gefurchtem Myzelgeflecht ( $\odot$ Abb.17 und $\odot$ Abb. 18). Einige Stämme beginnen mit glabrösem Wachstum und bilden erst nach mehreren Wochen Luftmyzel [1]. Die Kulturunterseite ist tiefrot oder weinrot $(\bullet$ Abb. 19$)$.

Mikromorphologie: Makroconidien sind selten, dünnwandig, zumeist lang und schmal, zylindrisch-keulenförmig und 2- bis 8zellig ( $\odot$ Abb.20).

Mikroconidien sind vereinzelt bis reichlich vorhanden, rundlich, oval oder birnenförmig und in Akladiumform angeordnet.

\section{Trichophyton mentagrophytes}

Perfektes Stadium: Arthroderma benhamiae

Synonym: Trichophyton granulosum, Trichophyton asteroides, Trichophyton mentagrophytes var. granulosum, Trichophyton mentagrophytes var. asteroides und viele andere

Trichophyton mentagrophytes ist ein bei Haus-, Wild- und Nagetieren relativ weit verbreiteter, zoophiler Dermatophyt und neben Trichophyton rubrum der aus menschlichem Nativmaterial am häufigsten isolierte Hautpilz. Befallen werden Haut, Haare und Nägel. Selten kommt die tiefe Trichophytie, wie z.B. das Kerion Celsi, vor [2].

Makromorphologie: Schnell wachsende, weißlich-cremefarbene, flache Kolonien mit fein- bis grobkörniger Oberfläche $(\bullet$ Abb.21, $\triangle$ Abb.22, $\odot$ Abb.24 und $\odot$ Abb.25). Die Kulturunterseite ist hellbraun bis braunrot.

Mikromorphologie: Makroconidien sind selten, dünnwandig, zylindrisch-keulenförmig und 3- bis 8-zellig ( $\bullet$ Abb.23).

Mikroconidien sind sehr zahlreich, rundlich bis piriform und zumeist in Botrytisform angeordnet.

Typisch für diese Pilzart ist vor allem das häufige Vorkommen von Spiralhyphen ( $\bullet$ Abb.26). Chlamydosporen werden ebenfalls gebildet und sind je nach Stamm selten bis reichlich vorhanden.

\section{Trichophyton mentagrophytes var. asteroides ${ }^{3}$}

Trichophyton mentagrophytes var. asteroides ist ein bei kleinen Nagetieren weit verbreiteter, zoophiler Dermatophyt. Durch die Aufnahme von Hamstern, Meerschweinchen und Kaninchen in die menschlichen Wohnbereiche ist dieser Pilz auch zunehmend häufiger als menschlicher Krankheitserreger in Erscheinung getreten.
Makromorphologie: Schnell wachsende, weiße, flache Kolonien mit puderig-körnigem Myzelgeflecht und sternförmig auslaufenden peripheren Hyphenbündeln ( $\bullet$ Abb.27). Die Kulturunterseite ist anfangs gelblich, später kupferrot bis rotbraun.

Mikromorphologie: Makroconidien sind zumeist selten, dünnwandig, an den Polen etwas zugespitzt oder stumpf-zylindrisch; die Zahl der Kammern schwankt zwischen 4 und 7 ( $\bullet$ Abb.28). Mikroconidien sind reichlich vorhanden, überwiegend rundlich, entlang den Hyphen oder in Traubenform angeordnet.

Spiralhyphen werden vor allem in älteren Kulturen sehr zahlreich gebildet ( $\bullet$ Abb.29).

\section{Trichophyton mentagrophytes var. quinckeanum ${ }^{4}$} Synonym: Achorion quinckeanum

Trichophyton mentagrophytes var. quinkeanum ist der Erreger des sogenannten „Mäusefavus“. Dieser, in Gegenden mit zahlreichen Mäusen häufiger vorkommende zoophile Dermatophyt wird gelegentlich auf den Menschen übertragen und verursacht eine Dermatophytose mit scutulumähnlicher Schuppung auf der glatten Haut, die früher als „Körperfavus“ bezeichnet wurde.

Makromorphologie: Schnell wachsende, reinweiße, flache Kolonien von puderig-samtiger oder flaumiger Konsistenz. Die Primärkulturen sind meist durch eine ausgeprägte Oberflächenfaltung gekennzeichnet $(\bullet$ Abb. 30).

Die Kulturunterseite ist anfangs tief gelb, später orange bis braun.

Mikromorphologie: Makroconidien sind sehr selten, dünnwandig, mehr oder weniger keulenförmig und überwiegend 4- bis 6zellig $(\bullet$ Abb.31).

Mikroconidien sind zahlreich vorhanden, rund, oval, birnenförmig oder länglich, mit zugespitzten Enden und in Akladiumform angeordnet ( $\bullet$ Abb.32). Fadenförmige Myzelfortsätze sind typisch für diese Variante [1].

\section{Trichophyton rubrum}

Synonym: Trichophyton purpureum, Epidermophyton rubrum

Trichophyton rubrum ist ein optimal an den Menschen angepasster Dermatophyt und weltweit mit Abstand der häufigste Erreger von Haut- und Nagelmykosen (Onychomykosen). Die Tendenz dieses anthropophilen Pilzes zur Chrinizität und seine Affinität zum Nagelkeratin verursachen erhebliche klinische Probleme [1].

Makromorphologie: Mäßig schnell wachsende, weißflaumige Kolonien mit erhabenem Zentrum und breitem, flachem, gelblich pigmentiertem Rand ( $\bullet$ Abb.33, $\odot$ Abb.34 und $\odot$ Abb.36). Die Kulturunterseite ist gelbrot bis rotbraun; manchmal mit ringförmig auftretendem Pigment ( $\bullet$ Abb. 35$)$.

Mikromorphologie: Makroconidien fehlen in der Regel (Ausnahme: granulöse Varianten) $(\bullet$ Abb. 37).

Mikroconidien sind sehr selten, birnenförmig und in Akladiumform angeordnet ( $\bullet$ Abb.38).

\footnotetext{
2 Trichophyton megninii wurde mit Trichophyton rubrum zusammengelegt.

3 Trichophyton mentagrophytes var. asteroides wurde mit Trichophyton mentagrophytes zusammengelegt.
}

\footnotetext{
${ }^{4}$ Trichophyton mentagrophytes var. quinckeanum wurde mit Trichophyton mentagrophytes zusammengelegt.
} 


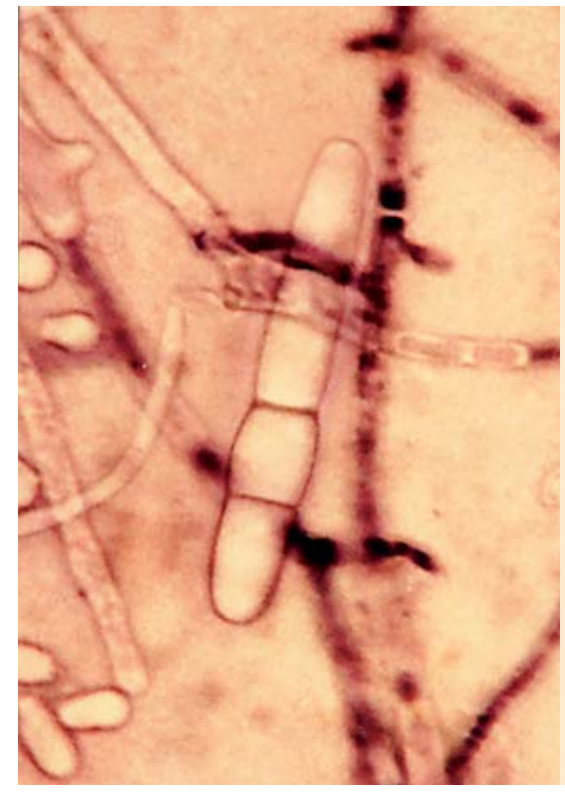

Abb.15 Makroconidie von Trichophyton interdigitale (stark vergrößert).
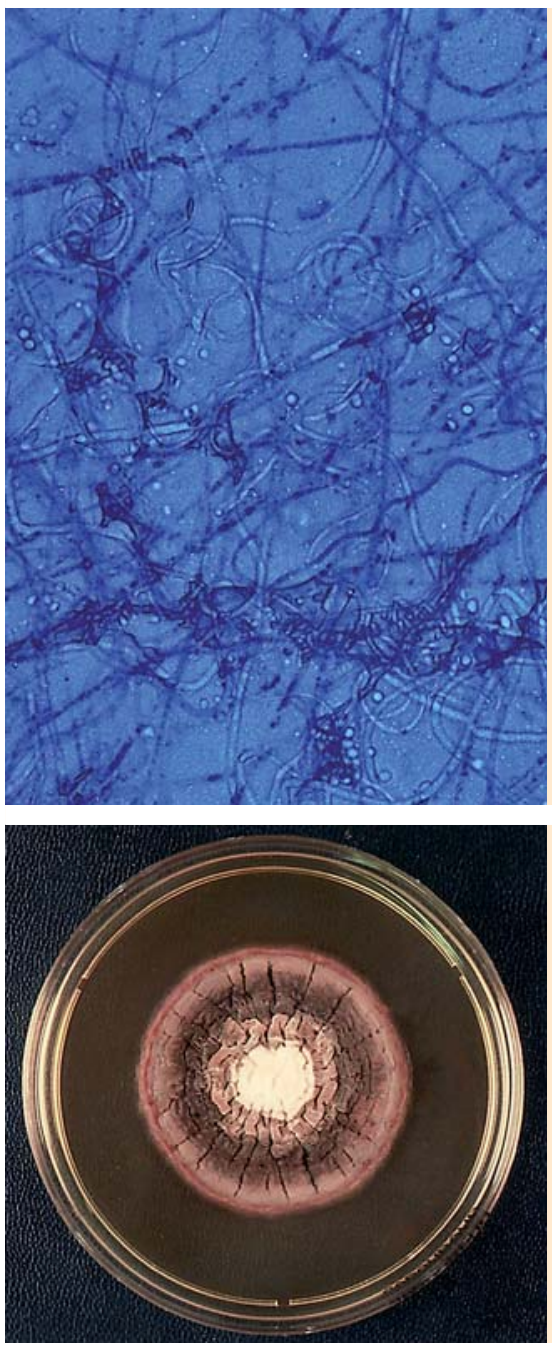

Abb.16 Spiralhyphen und Mikroconidien von Trichophyton interdigitale (stark vergrößert).

Abb.17 Trichophyton megninii auf KimmigAgar. 30 Tage alte, rosa pigmentierte Monokultur mit samtig-flaumiger, unregelmäßig radiär gefurchter Oberfläche.

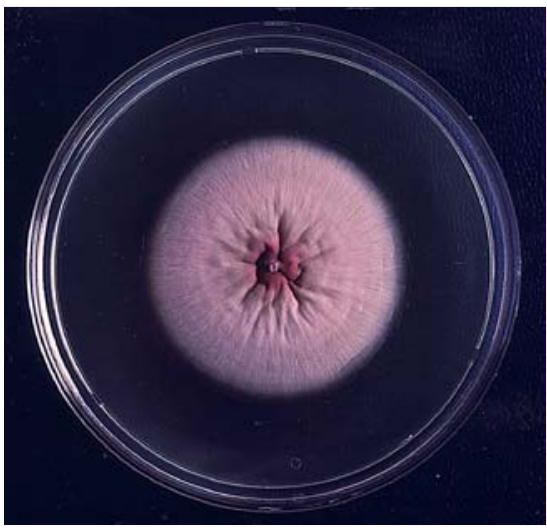

Abb.18 Trichophyton megninii auf Sabouraud-Glucose (2\%)-Agar. 35 Tage alte, rot-violett pigmentierte Monokultur mit kurzflaumigem, zentral gefurchtem Myzelgeflecht.

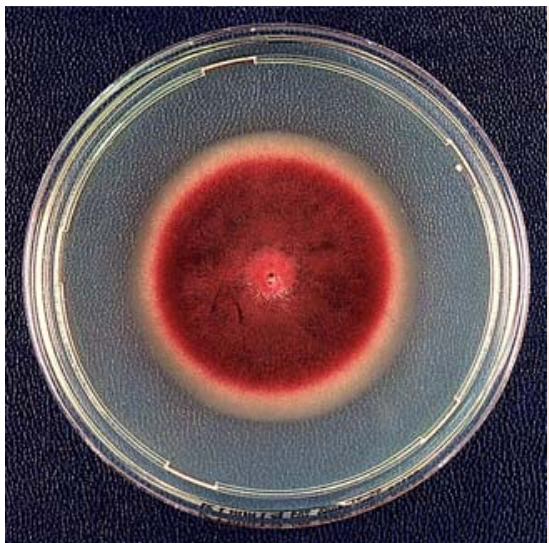

Abb.19 Weinrot pigmentierte Kulturunterseite von Trichophyton megninii.

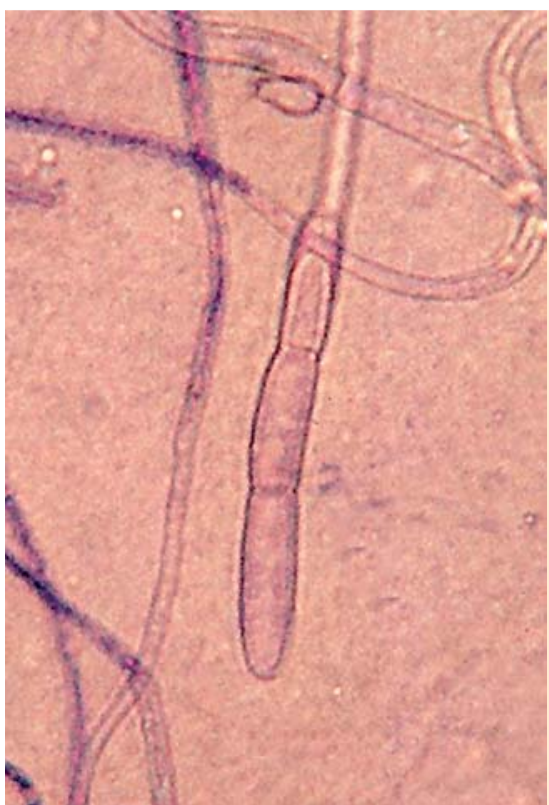

Abb.20 Makroconidie von Trichophyton megninii (stark vergrößert).

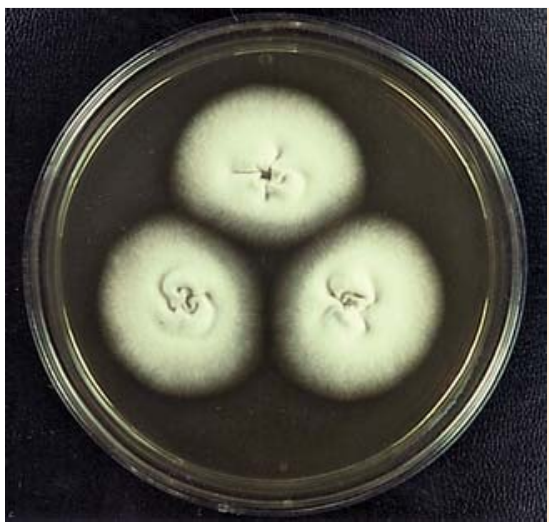

Abb.21 Trichophyton mentagrophytes auf Kimmig-Agar. 19 Tage alte, weißlich-cremefarbene Drillingskultur mit feinkörniger bis gipsiger Oberfläche. 

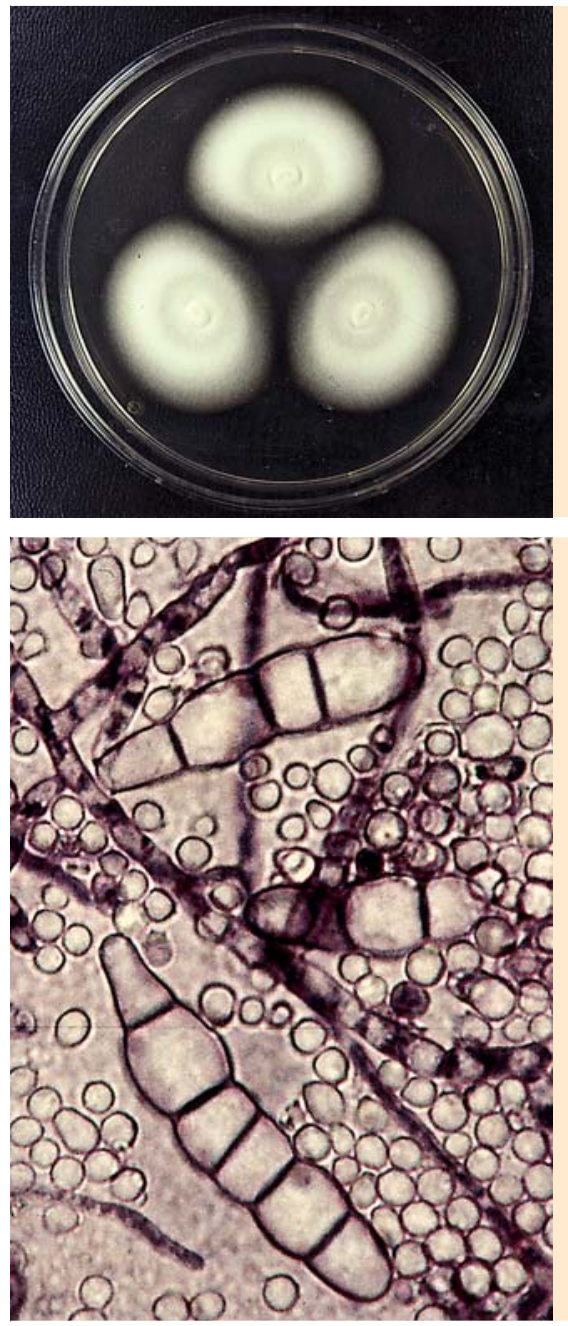

Abb. 23 Makroconimentagrophytes (stark vergrößert).

Abb.22 Trichophyton mentagrophytes auf Sabouraud-Maltoseweiße Drillingskultur mit feinkörniger Oberfläche.
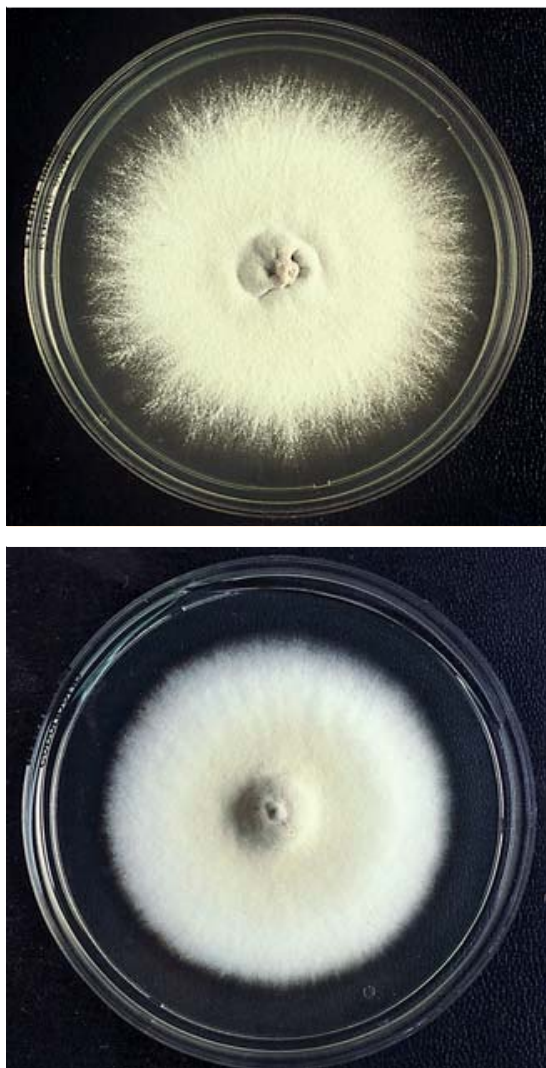

Abb.24 Trichophyton mentagrophytes auf Sabouraud-GlukoseAgar (2\%). 22 Tage alte, cremefarbene Monokultur mit feinkörniger bis gipsiger Oberfläche.

Abb.25 Trichophyton mentagrophytes auf Mycosel-Agar. 24 Tage alte, weißlich-cremefarbene Monokultur mit feinkörniger Ober-

fläche. dien von Trichophyton Agar. 21 Tage alte,
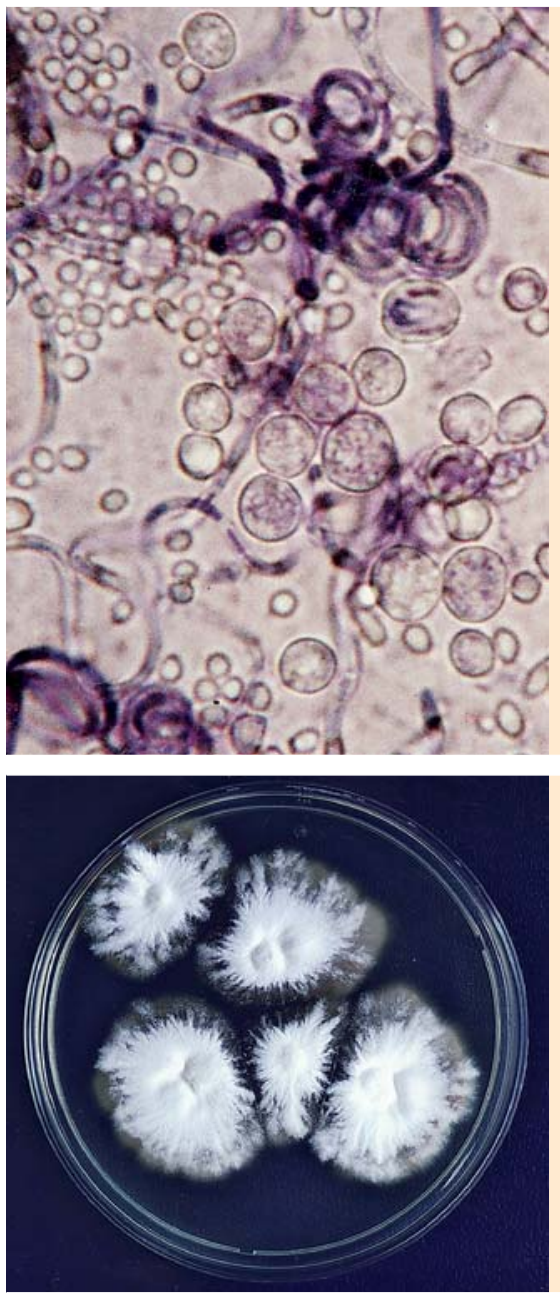

Abb.27 Trichophyton mentagrophytes var. asteroides auf Sabouraud-Gluksose-Agar (2 \%). 19 Tage alte, weiße Reinkultur mit puderigkörniger Oberfläche und sternförmig auslaufenden peripheren Hyphenbündeln.

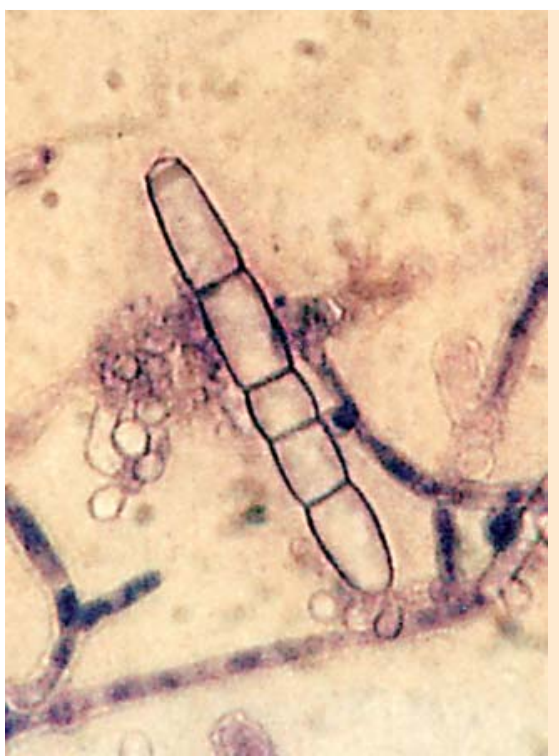

Abb. 28 Makroconidie von Trichophyton mentagrophytes var. asteroides (stark vergrößert). 


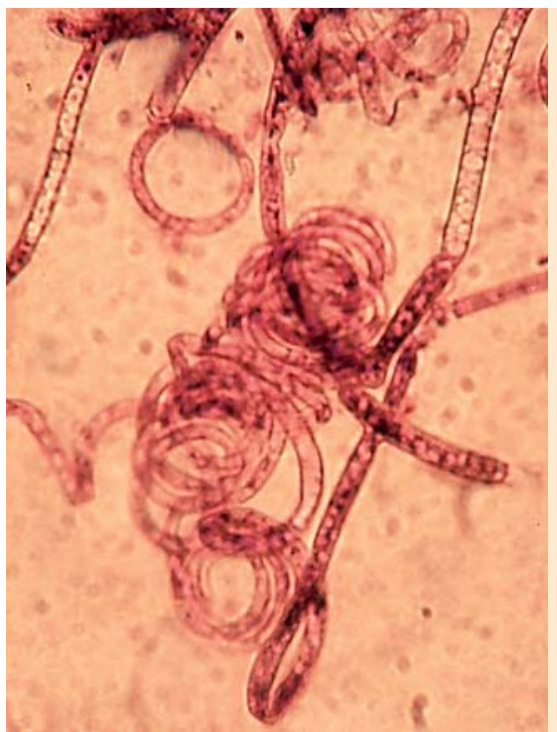

Abb.29 Spiralhyphe von Trichophyton mentagrophytes var. asteroides (stark vergrößert).
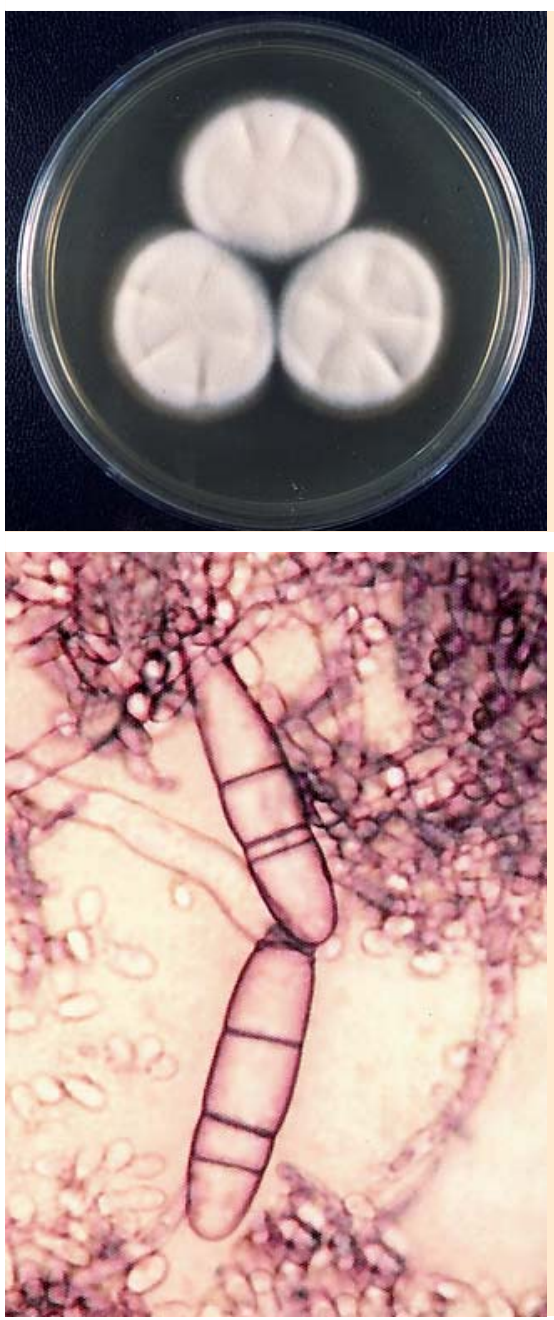
mentagrophytes var. quinckeanum auf Kimradiär gefurchte Drillingskultur mit weißer, dichter, puderig-samtiger Oberfläche.

Abb. 31 Makro- und Mikroconidien von Trichophyton mentaanum (stark vergrößert).

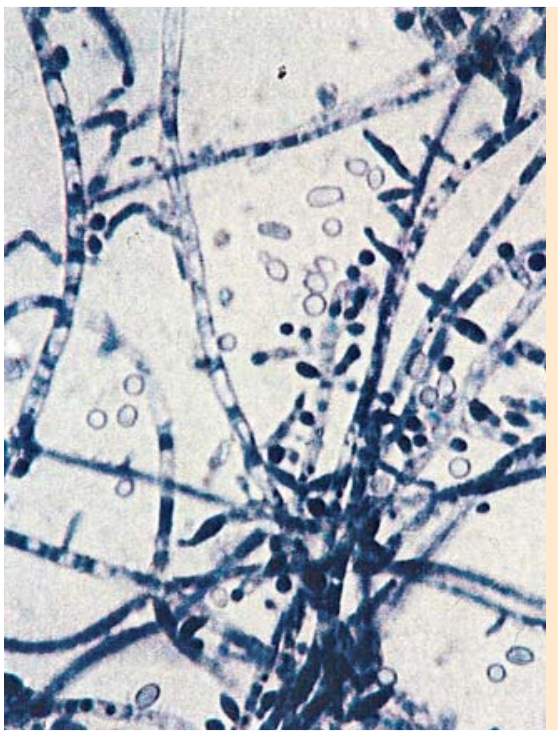

Abb.32 Mikroconidien von Trichophyton mentagrophytes var. quinckeanum (stark vergrößert).

Abb. 30 Trichophyton mig-Agar. 12 Tage alte, grophytes var. quincke-

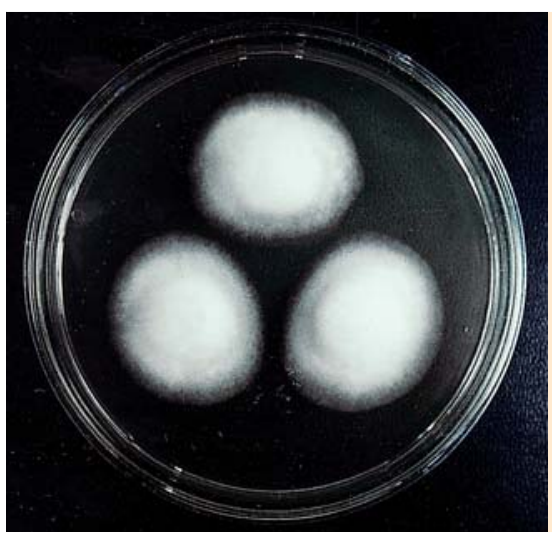

Abb.33 Trichophyton rubrum auf SabouraudMaltose-Agar. 30 Tage alte, weiße Drillingskultur mit hutförmigen Kolonien.

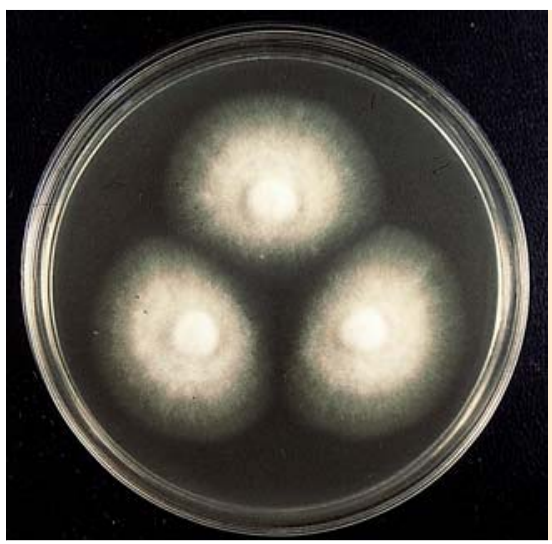

Abb.34 Trichophyton rubrum auf KimmigAgar. 25 Tage alte, cremefarbene Drillingskultur mit zentraler Erhabenheit und breitem, flachem Rand.

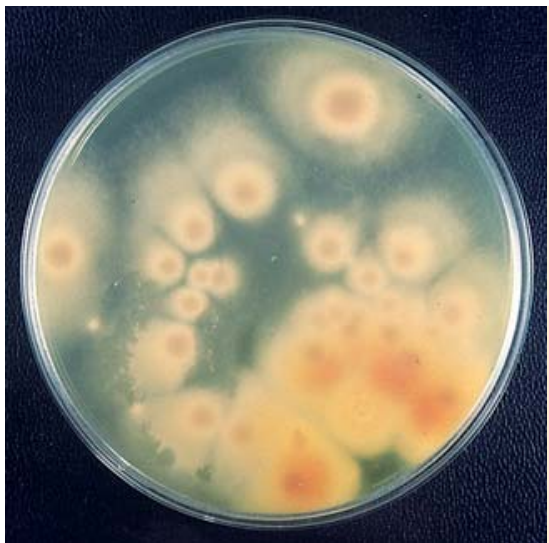

Abb.35 Rotgelb pigmentierte Kulturunterseite von Trichophyton rubrum. 


\section{Trichophyton schoenleini}

Synonym: Oidium schoenleinii, Achorion schoenleinii

Dieser, vor allem in Afrika und Südosteuropa vorkommende anthropophile Dermatophyt ist der klassische Favus-Erreger. Die Infektionen manifestieren sich vorwiegend am behaarten Kopf. Charakteristisch ist die "Scutula-Bildung“ mit Haarbefall und nachfolgendem narbigen Haarausfall. Mitunter können auch die Haut und die Nägel befallen sein. Im Gegensatz zum Favus des behaarten Kopfes sieht man dann zwar auch entzündliche Veränderungen mit Schuppen- und Krustenbildung, jedoch keine narbige Atrophie [3].

Makromorphologie: Langsam wachsende, grau-weiße, zerebriforme, gefaltete und tief gefurchte Kolonien mit glatter, feuchter, wachsartiger Oberfläche ( $\bullet$ Abb. 39 und $\odot$ Abb. 40). Die Kulturunterseite ist farblos.

Mikromorphologie: Makroconidien fehlen in der Regel. Mikroconidien sind sehr selten, 1-, 2- und 3-zellig; man erhält sie auf feuchten Reiskörnern. Diagnostisch wegweisend sind dichotome Verzweigungen ( $\bullet$ Abb.41), „Geweih- und Kronleuchter-Hyphen" sowie terminale und interkalare Chlamydosporen. Trichophyton schoenleinii wächst gut bei $35-37^{\circ} \mathrm{C}$. Woodlicht: graugelbe bis grauweiße Fluoreszenz-Färbung.

\section{Trichophyton soudanense ${ }^{5}$}

Dieser anthropophile, in Europa immer häufiger als Einwanderungspilz vorkommende Dermatophyt befällt bevorzugt den behaarten Kopf und verursacht eine Dermatophytose mit meist nur oberflächlichen, wenig ausgeprägten entzündlichen Reaktionen. Man findet in den leicht abbrechenden Haaren aus den diskret schuppenden Herden endotrich wachsende Pilzfäden mit rascher großzelliger Arthrosporenbildung [1].

Makromorphologie: Langsam wachsende, intensiv gelbe oder aprikosenfarbene, flache Kolonien, mit knopfartigem Zentrum und dichter, samtähnlicher, gefurchter Oberfläche. Der Kolonienrand ist von sehr feinen, strahlenförmig auslaufenden, peripheren Hyphenbündeln umgeben ( $\bullet$ Abb.42 und $\odot$ Abb.43). Die Kulturunterseite ist weißgelb bis karottenrot.

Mikromorphologie: Makroconidien fehlen. Mikroconidien sind selten, 1- und 2-zellig, überwiegend birnenförmig und in Akladiumform angeordnet.

Charakteristische Mikrostrukturen sind Hyphen mit gegenläufigen Seitenverzweigungen (Crossing-Over-Phänomen) ( $\bullet$ Abb. 44). Typisch für diese Pilzart ist außerdem das zahlreiche Vorkommen von Chlamydosporen und ein frühzeitiger Zerfall der Hyphen in Arthrosporen.

\section{Trichophyton terrestre}

Perfekte Stadien: Arthroderma quadrifidum, Arthroderma lenticularum, Arthroderma insingulare

Trichophyton terrestre ist ein nahezu apathogener, geophiler Dermatophyt.

Makromorphologie: Schnell wachsende, weißlich-cremefarbene Kolonien, mit feinkörniger bis gipsiger Oberfläche, die stellenweise von zartem Flaum überzogen wird $(\bullet \mathrm{Abb} .45$ und $\odot \mathbf{A b b}$.

\footnotetext{
${ }^{5}$ Trichophyton soudanense wurde mit Trichophyton violaceum zusammengelegt.
}

46). Die Kulturunterseite ist beige, manchmal gelbbraun bis rotbraun. Intensiver, charakteristischer Geruch.

Mikromorphologie: Typisch für diese Pilzart sind zahlreiche birnenförmige bis längliche, in Akladiumform angeordnete Mikroconidien, die kontinuierlich über 2- und 3-zellige Intermediärformen in 3- bis 5-fach septierte, dünnwandige, meist zylindrischkeulenförmige Makroconidien übergehen ( $\bullet$ Abb.47). Andere charakteristische Mikrostrukturen, wie Spiralhyphen und Chlamydosporen, werden gelegentlich gebildet.

\section{Trichophyton tonsurans}

Synonym: Trichophyton epilans, Trichophyton crateriforme, Trichophyton flavum, Trichophyton sulfureum u.a.

Trichophyton tonsurans ist ein, vor allem in den USA (Chicago), relativ weit verbreiteter anthropophiler Dermatophyt mit besonderer Affinität zum Haar. Die Infektionen manifestieren sich vorwiegend im Bereich des Capillitiums und verursachen eine Dermatophytose mit massiver Hyphen- und Sporenbildung innerhalb des Haarschaftes. Die befallenen Haare verlieren ihre Elastizität und brechen wenige Millimeter oberhalb des Hautniveaus ab. Die mit zahlreichen Sporen angereicherten Haarstümpfe erscheinen als „dicke schwarze Punkte“ auf der Kopfhaut und sind typisch für das klinische Bild (black dot) dieser Pilzerkrankung [4]. Aufgrund des endotrichen Wachstums treten meist nur geringgradig ausgeprägte Entzündungserscheinungen auf. Dieser Hautpilz kann jedoch auch gelegenlich die mit Kerionbildung einhergehende tiefe Trichophytie, vor allem im Bartbereich älterer Patienten, hervorrufen.

Trichophyton tonsurans tritt in 4 verschiedenen Varianten auf, nämlich „crateriforme“, „sabouraudii“, „epilans“ und „sulfureum".

Weltweit dominiert die Variante sulfureum. Sie ist als Erreger der Tinea corporis gladiatorum im Kampf- und Fitnesssport verbreitet [5].

Das Auftreten von Varianten ist von bestimmten, den Pilzen innewohnenden Faktoren abhängig, die sie befähigen, abweichende, vom Nährboden unabhängige Wuchsformen zu bilden. Die morphologischen Varianten können mitunter erhebliche diagnostische Probleme verursachen, denn es kommt leicht zu Verwechslungen mit anderen Dermatophytenarten, wie z.B. mit Trichophyton rubrum, Trichophyton mentagrophytes oder Epidermophyton floccosum [6].

Makromorphologie: Mäßig schnell wachsende, weiße, gelbe, rote oder rotbraune, flache Kolonien, mit zentraler, zerklüfteter Delle und kurzflaumiger, samt- oder wildlederähnlicher Oberfläche ( $\bullet$ Abb.48-50). Der Kolonienrand ist glatt oder von sehr feinen, strahlenförmig auslaufenden Hyphenbündeln umgeben. Die Kulturunterseite ist rötlich-braun, braun oder mahagonifarben.

Mikromorphologie: Makroconidien sind selten, dünnwandig, überwiegend zylindrisch keulenförmig und 2- bis 4-, selten bis 9-zellig ( $\bullet$ Abb.51).

Mikroconidien sind sehr zahlreich, von unterschiedlicher Form und Größe, lateral an den Hyphen oder in einfacher Traubenform angeordnet ( $\bullet$ Abb. 52).

Chlamydosporen $(\bullet$ Abb. 53) und Spiralhyphen werden ebenfalls gebildet und sind je nach Stamm und Alter der Pilzkultur in unterschiedlicher Anzahl vorhanden. 


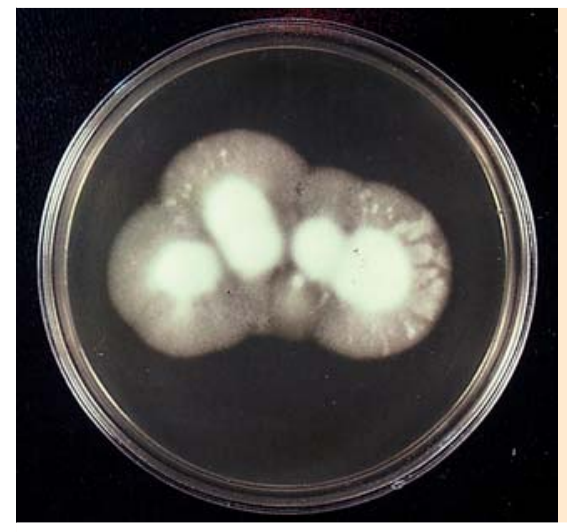

Abb.36 23 Tage alte, watteähnliche Primärkultur von Trichophyton rubrum auf SabouraudMaltose-Agar.

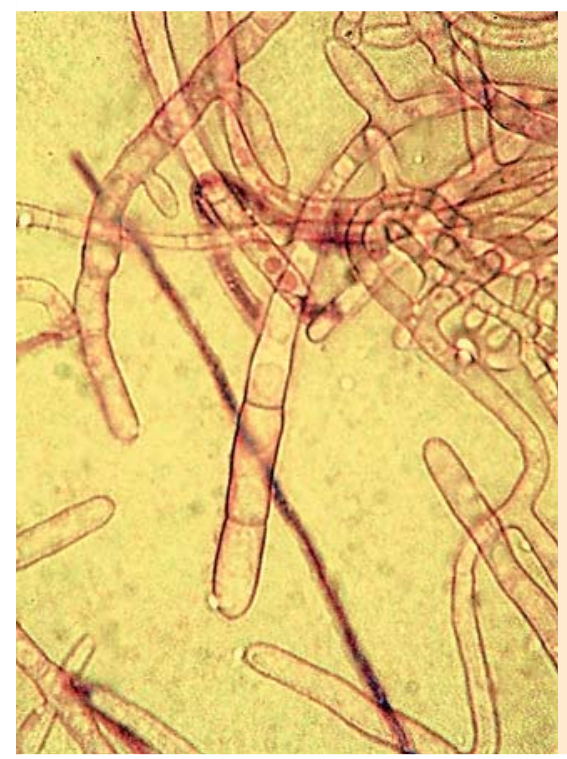

Abb.37 Makroconidie von Trichophyton rubrum (stark vergrößert).

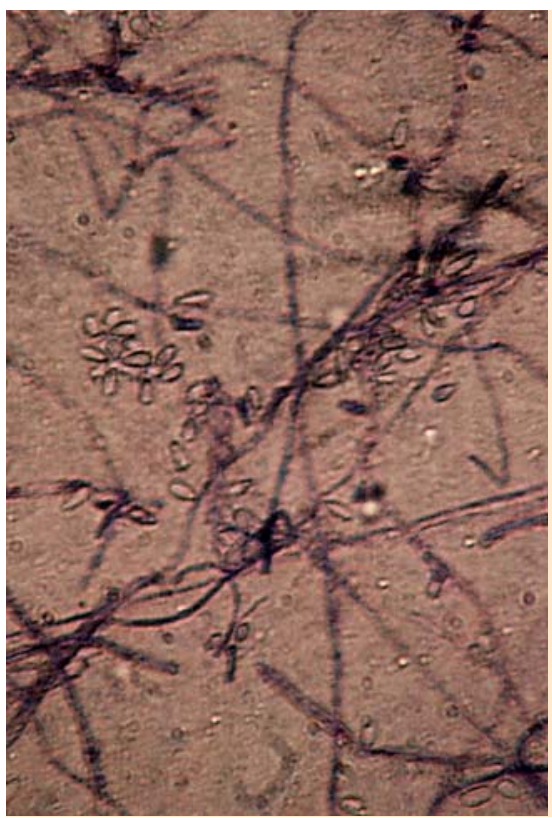

Abb. 38 Mikroconidien von Trichophyton rubrum (stark vergrößert).

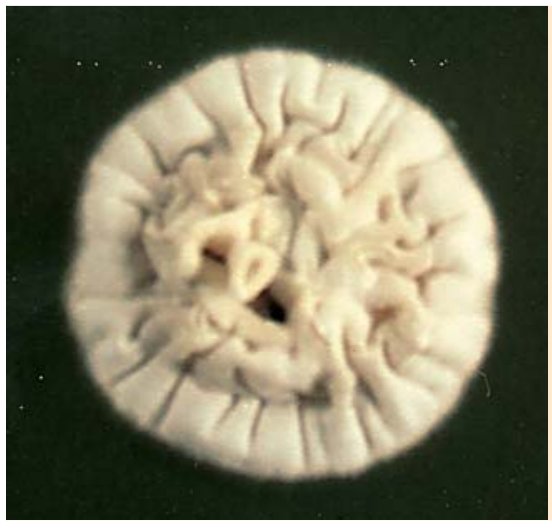

Abb.39 Trichophyton schoenleinnii auf Kimmig-Agar. 32 Tage alte weißliche, tief gefurchte Monokultur mit wachsartiger Oberfläche (stark vergrößert).

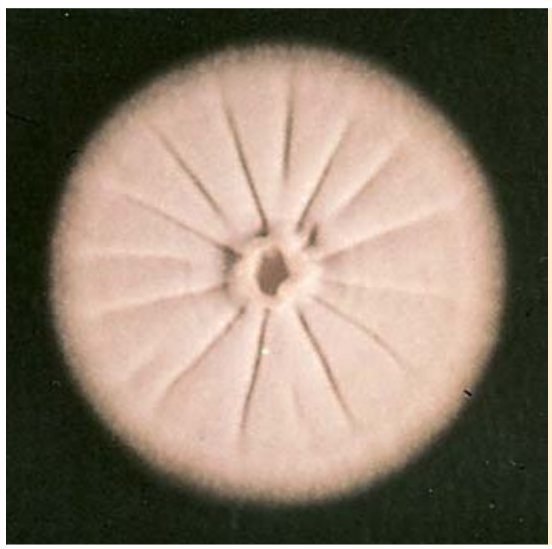

Abb.40 Trichophyton schoenleinii auf Sabouraud-Maltose-Agar. 32 Tage alte, weißliche, radiär gefurchte Monokultur mit kurzflaumiger, fast glatter Oberfläche (stark vergrößert).

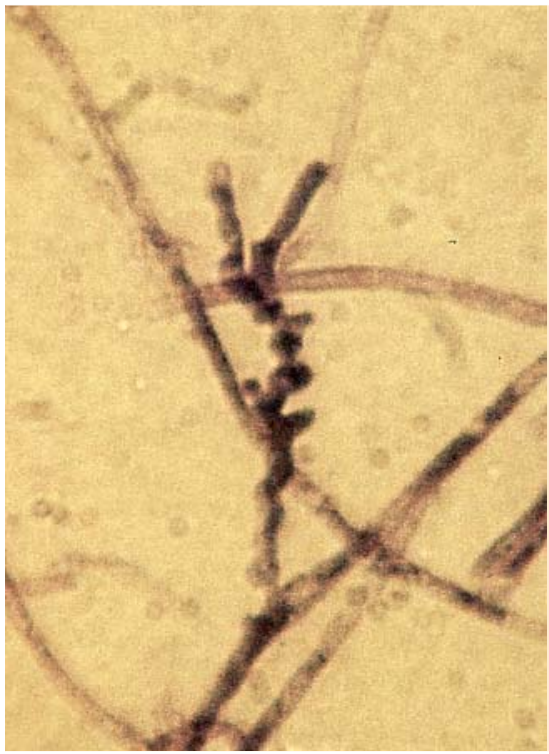

Abb.41 Dichotome Hyphenverzweigung von Trichophyton schoenleinii (stark vergrößert).

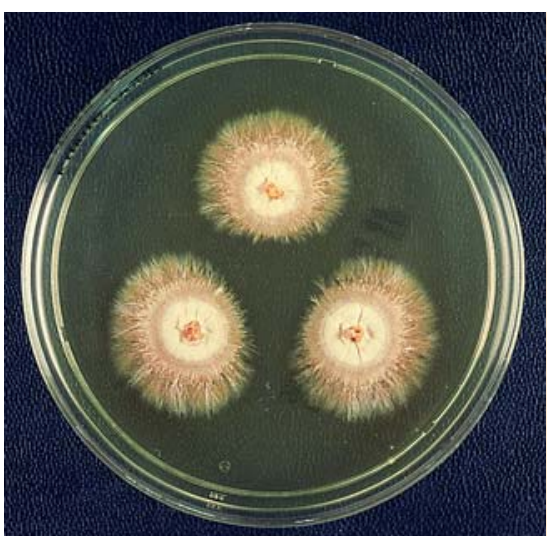

Abb.42 Trichophyton soudanense auf Kimmig-Agar. 30 Tage alte, flache, aprikosenfarbene Drillingskultur mit strahlenförmigem Randsaum. 

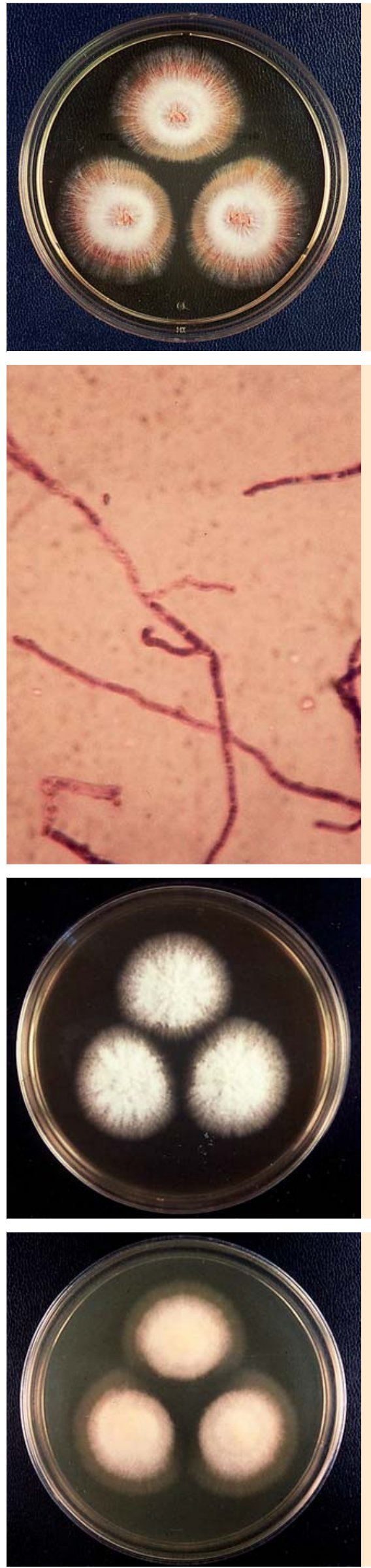

Abb.43 30 Tage alte, flache, aprikosenfarbene Drillingskultur mit strahlenförmig auslaufenden Hyphenbündeln eines anderen Sammlungsstammes von Trichophyton soudanense auf KimmigAgar.

Abb.44 Vor-und rückwärts wachsende Seitenhyphen von Trichophyton soudanense (stark vergrößert).

Abb.45 Trichophyton terrestre auf KimmigAgar. 7 Tage alte, weiße Drillingskultur mit grobkörniger bis gipsiger Oberfläche.

Abb.46 Trichophyton terrestre auf Sabouraud-Maltose-Agar. 9 Tage alte, weiße Drillingskultur mit flaumigpuderiger Oberfläche und abgesetztem Randsaum.

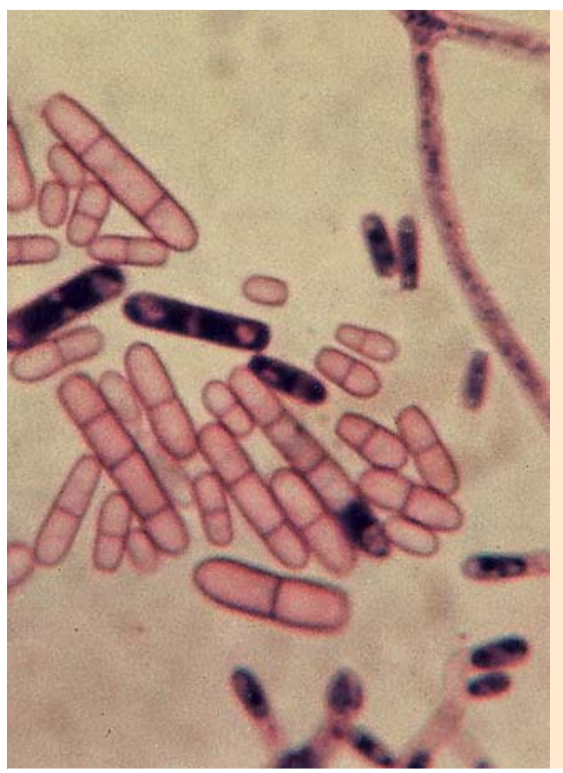

Abb. 47 Makroconidien, 2- und 3-zellige Intermediärformen und Mikroconidien von Trichoton terrestre (stark vergrößert).

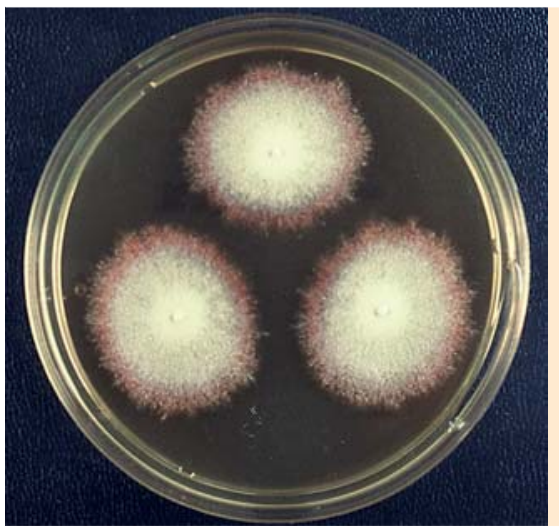

Abb.48 Trichophyton tonsurans auf Sabouraud-Glukose-Agar (2\%). 27 Tage alte Drillingskultur mit gelblicher, puderig-körniger, im Zentrum etwas dichterer Oberfläche und strahlenförmig auslaufenden, rotbraunen Hyphenbündeln.

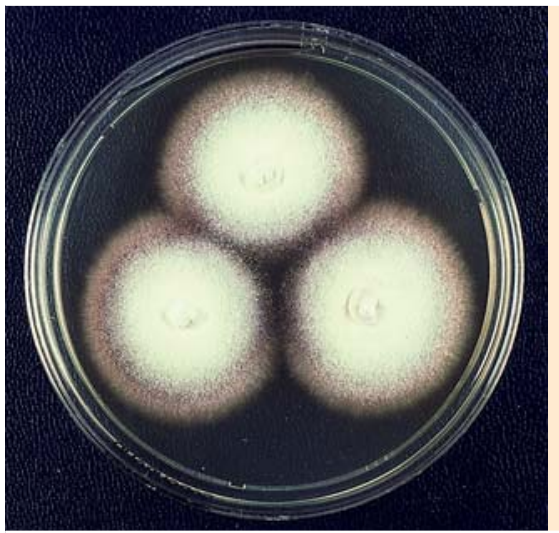

Abb.49 27 Tage alte Drillingskultur mit beigefarbener, puderiger Oberfläche und abgesetztem hellbraunem Randsaum eines anderen Sammlungsstammes auf Sabouraud-Glukose-Agar (2\%).

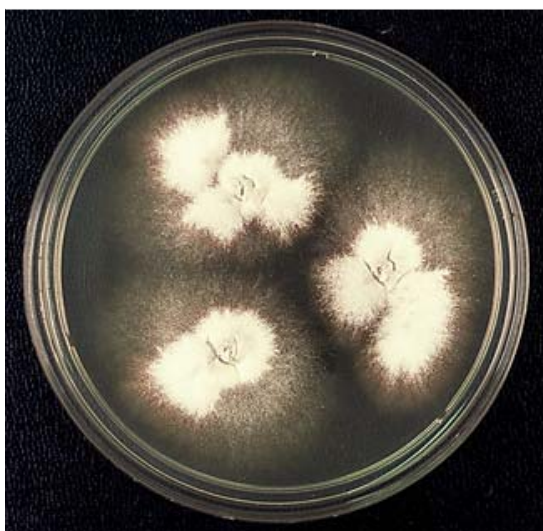

Abb.50 Trichophyton tonsurans auf KimmigAgar. 31 Tage alte Reinkultur mit beigefarbener, puderig-körniger, im Randbereich gelbbraun pigmentierter Oberfläche. 


\section{Trichophyton verrucosum}

Synonym: Trichophyton album, Trichophyton ochraceum, Trichophyton discoides

Trichophyton verrucosum ist der Erreger der „Rinder-, Kälberoder Glatzflechte“. Dieser, vor allem in ländlichen Gebieten mit intensiver Viehzucht gehäuft vorkommende zoophile Dermatophyt wird durch direkten oder indirekten Kontakt mit infizierten Tieren auf den Menschen übertragen und verursacht vorwiegend im Bereich des Kapillitiums, der Bartregion sowie der Unterarme eine follikuläre, entzündliche Dermatophytose. Die Zerstörung der Haarfollikel durch invasives Pilzwachstum und Entzündungsreaktion mündet in einer narbigen Alopezie [7].

Makromorphologie: Sehr langsam wachsende, unregelmäßig gefurchte, warzenartig gebuckelte oder scheibchenförmige Kolonien, von fester Konsistenz, mit weißer bis gelbbrauner, kurzflaumiger, fast glatter Oberfläche ( $\bullet$ Abb.54 und $\odot$ Abb.55). Die Kulturunterseite ist farblos.

Mikromorphologie: Makroconidien fehlen in der Regel. Mikroconidien sind keulenförmig und erscheinen nur im Thiamin-angereicherten Medium ( $\bullet$ Abb.57) [8]. Typisch für diese Pilzart sind Arthrosporen-Hyphen ( $\bullet$ Abb.56) sowie terminale und interkalare Chlamydosporen, die vor allem in älteren Kulturen sehr zahlreich gebildet werden.

Cave!

Einige Stämme von Trichophyton verrucosum wachsen erst bei $35-37^{\circ} \mathrm{C}$.

\section{Trichophyton violaceum}

Synonym: Trichophyton glabrum, Achorion violaceum, Trichophyton soudanense

Trichophyton violaceum ist ein in Nord- und Zentralafrika weit verbreiteter, antropophiler Dermatophyt und Erreger einer meist chronischen Dermatophytose des behaarten Kopfes und/oder der übrigen Haut mit gelegentlichem Befall des zugehörigen lymphatischen Gewebes.

Makromorphologie: Langsam wachsende, rosa bis rotviolette Kolonien mit glattem, wachsartigem, stark gefaltetem Myzelgeflecht ( $\odot$ Abb.58, $\odot$ Abb.59, $\odot$ Abb.61 und $\odot$ Abb. 62). Trichophyton violaceum wächst durchaus nicht immer mit der typisch kleinen, violett gefärbten Kolonie, es können sich weiße, flaumige Varianten bilden, die Trichophyton mentagrophytes täuschend ähnlich sehen [9]. Die Kulturunterseite ist dunkelviolett.

Mikromorphologie: Makroconidien sind sehr selten, dünnwandig, von unterschiedlicher Form und Größe; die Zahl der Kammern schwankt zwischen 3 und 5.

Mikroconidien sind selten, rundlich, oval oder birnenförmig und gelegentlich auf Trypsin- oder Thiamin-angereichertem Sabouraud-Dextrose-Agar zu finden.

Terminale und interkalare, dickwandige Chlamydosporen sind vor allem in älteren Kulturen sehr zahlreich vorhanden ( $\bullet$ Abb. 60 und $\odot$ Abb.63).

\section{Gattung Epidermophyton}

$\nabla$

\section{Epidermophyton floccosum}

Synonym: Acrothecium floccosum, Epidermophyton inquinale, Epidermophyton cruris

Epidermophyton floccosum ist die einzige humanpathogene Pilzart der Gattung Epidermophyton. Dieser anthropophile Dermatophyt verursacht vor allem Mykosen im Inquinal- und Zehenbereich (mit und ohne Nagelbeteiligung). Andere Körperbereiche werden dagegen relativ selten befallen.

Makromorphologie: Mäßig schnell wachsende, ocker bis olivfarbene, oft zentral gefurchte Kolonien mit feinfransiger, samtiger, wildlederähnlicher oder puderiger Oberfläche, die bei pleomorphen Kulturen weiße, watteähnliche Sektoren aufweist (flaumige Degeneration). Der Kolonienrand ist glatt oder von strahlenförmig auslaufenden peripheren Hyphenbündeln umgeben (๑ Abb.64, $\odot$ Abb. 65, $\odot$ Abb.67 und $\odot$ Abb. 68).

Die Kulturunterseite ist beige-braun pigmentiert.

Mikromorphologie: Makroconidien sind selten bis zahlreich, glatt- und dünnwandig, keulenförmig, 2- bis 4-zellig, einzeln oder in Haufen vorkommend ( $\bullet$ Abb.66). Mikroconidien sind nicht vorhanden. Chlamydosporen werden sowohl terminal als auch interkalar, vor allem in älteren Kulturen auffällig häufig gebildet ( $\bullet$ Abb.69).

\section{Gattung Microsporum}

$\nabla$

Die Gattung Microsporum umfasst 15 Arten. Von humanmedizinischer Bedeutung sind insbesondere Microsporum audouinii, Microsporum canis und Microsporum gypseum.

\section{Microsporum audouinii}

Synonym: Microsporum velveticum, Microsporum tardum, Microsporum depauperatum, Sabouraudites audouinii

Microsporum audouinii ist der Erreger der klassischen „Mikrosporie der Kinderköpfe“. Dieser anthropophile Dermatophyt verursacht im Bereich des Kapillitiums meist rundliche, weißlichgraue, schuppende Pilzinfektionen. Die befallenen Haare, die oft wie ein „Sack voller Nüsse“ aussehen, werden spröde und brechen kurz über dem Hautniveau ab. Der Verlauf der Infektion ist in der Regel entzündungsarm mit deutlicher Tendenz zur Chronizität [1].

Makromorphologie: Mäßig schnell wachsende, weiße, grauweiße oder leicht bräunliche, flache Kolonien, von samtiger, feinflaumiger oder watteähnlicher Konsistenz und manchmal angedeutetem radiärfaltigem Oberflächenprofil $(\bullet \mathbf{A b b . 7 0}$ und - Abb. 71). Die Kulturunterseite ist beige, orange oder rötlichbraun.

Mikromorphologie: Makroconidien sind selten, dick- und rauwandig, unregelmäßig spindelförmig und 1- bis 7-fach septiert. Mikroconidien sind ebenfalls selten, keulenförmig und in Akladiumform angeordnet ( $\bullet$ Abb.73). Diagnostisch wegweisend für diese Pilzart sind Tennisschläger- und „Kammzinken“-Hyphen sowie zahlreiche, vor allem terminal gebildete Chlamydosporen ( $\bullet$ Abb. 72). Woodlicht: grüne Fluoreszenz-Färbung. 


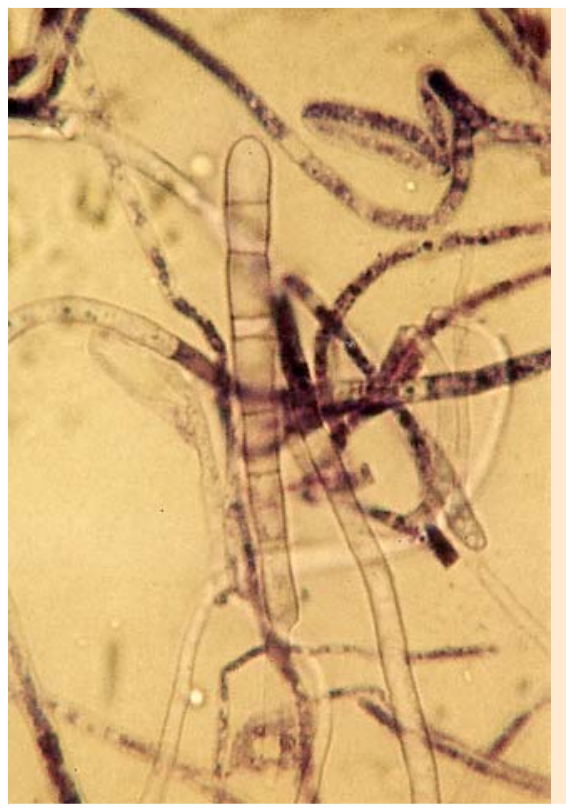

Abb.51 Makroconidie von Trichophyton tonsurans (stark vergrößert).
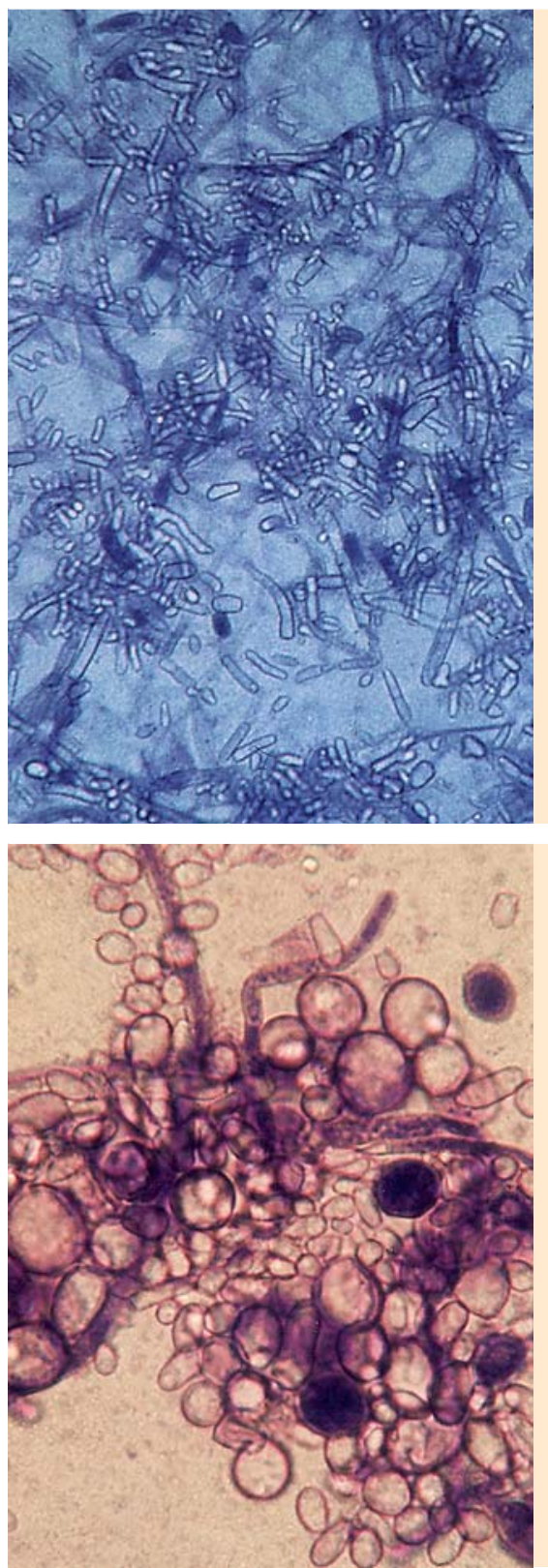

Abb.52 Mikroconidien von unterschiedlicher Form und Größe von Trichophyton tonsurans (stark vergrößert).

Abb.53 Chlamydosporen und Mikroconidien von Trichophyton tonsurans (stark vergrößert).

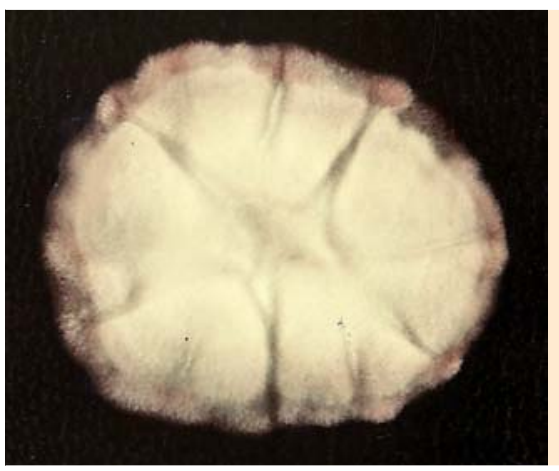

Abb.54 Trichophyton verrucosum auf Kimmig-Agar. 36 Tage alte, weißlich-cremefarbene, in der Peripherie leicht bräunliche Monokultur mit samtiger, gefurchter Oberfläche.

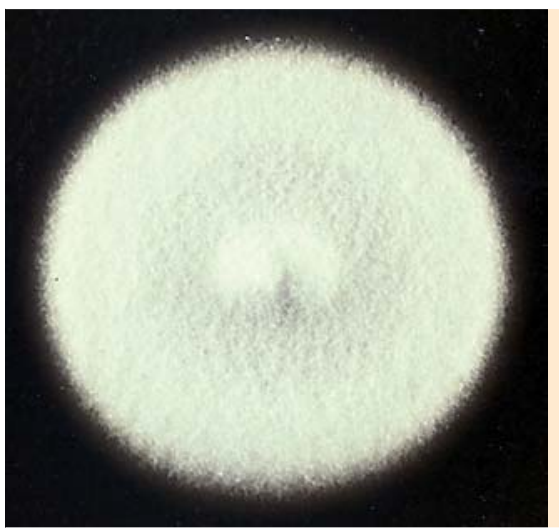

Abb.55 36 Tage alte, weiße Monokultur mit kurzflaumiger Oberfläche und warzenartiger, zentraler Knopfbildung eines anderen Sammlungsstammes von Trichophyton verrucosum auf KimmigAgar.
Abb.57 Mikroconidien von Trichophyton verrucosum (stark vergrößert).

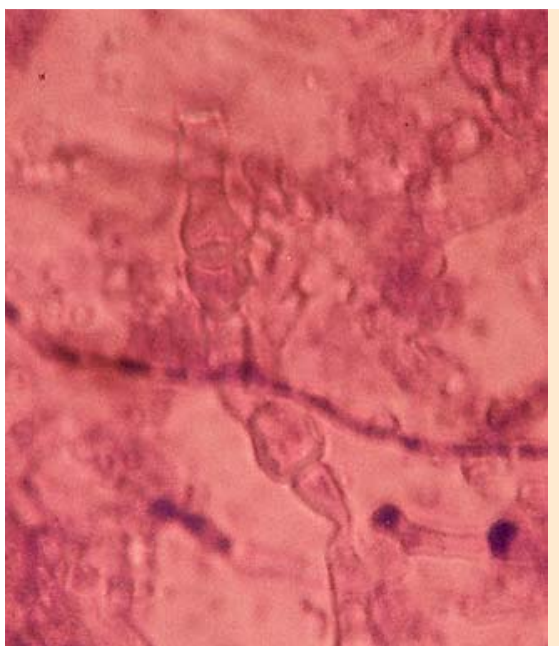

Abb.56 Arthrosporen-Hyphe von Trichophyton verrucosum (stark vergrößert).

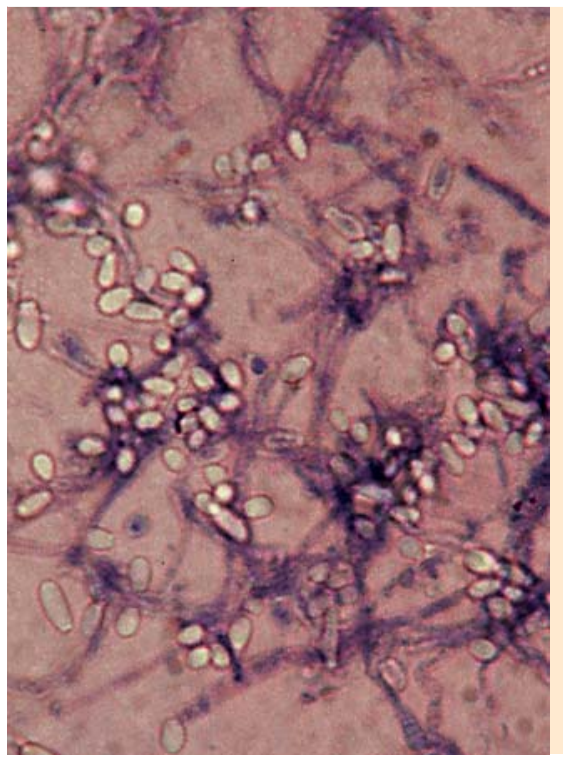



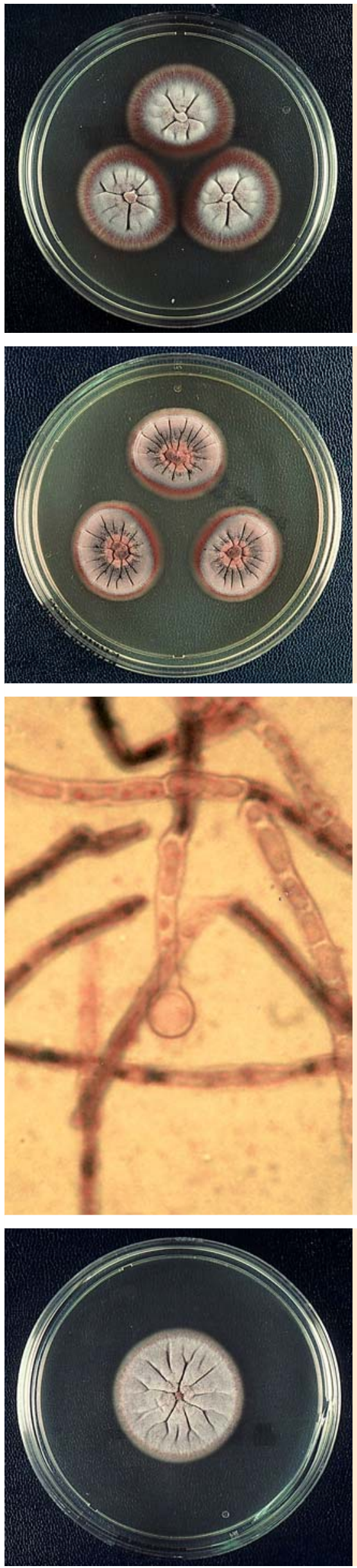

Abb.58 Trichophyton violaceum auf Sabouraud-Glukose-Agar (2\%). 27 Tage alte Drillingskultur mit samtiger, radiär gefurchter, im Randbereich violett pigmentierter Oberfläche.

Abb.59 Trichophyton violaceum auf KimmigAgar. 27 Tage alte, samtige, radiär gefurchte Drillingskultur mit schmalem, violett pigmentiertem Randsaum.

Abb.60 Terminal gebildete Chlamydospore von Trichophyton violaceum (stark vergrößert).

Abb.61 Trichophyton violaceum auf KimmigAgar. 31 Tage alte Monokultur mit samtiger bis kurzflaumiger, unregelmäßig gefurchter Oberfläche.

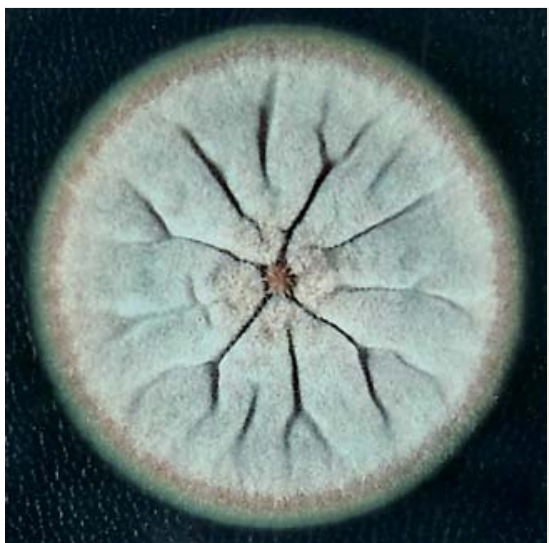

Abb.62 Dieselbe Monokultur von Trichophyton violaceum (s. Abb. 61) stark vergrößert.

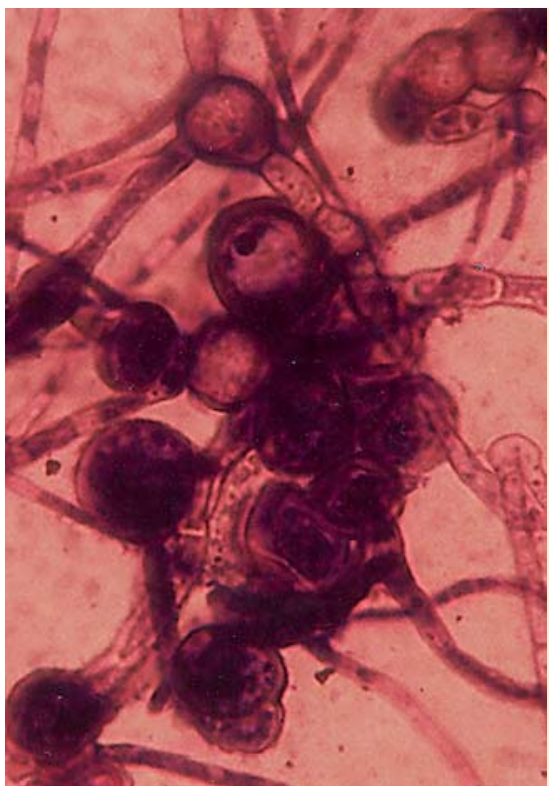

Abb.63 Chlamydosporen von Trichophyton violaceum (stark vergrößert).

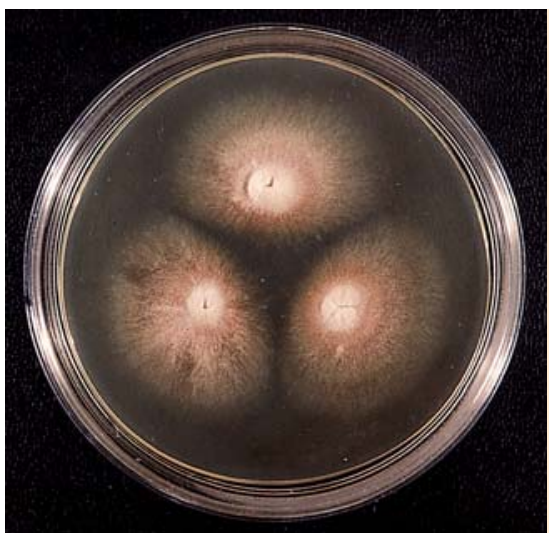

Abb.64 Epidermophyton floccosum auf Sabouraud-GlukoseAgar (2\%). 28 Tage alte Drillingskultur mit sehr feinem, kurzflaumigem Luftmyzel.

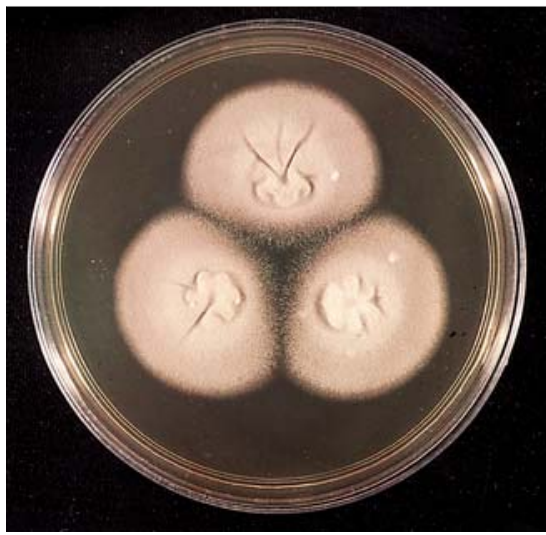

Abb.65 Epidermophyton floccosum auf Kimmig-Agar. 22 Tage alte Drillingskultur mit samtiger, zentral gefurchter Oberfläche und typischen (kleinen) pleomorphen Flaumflöckchen. 


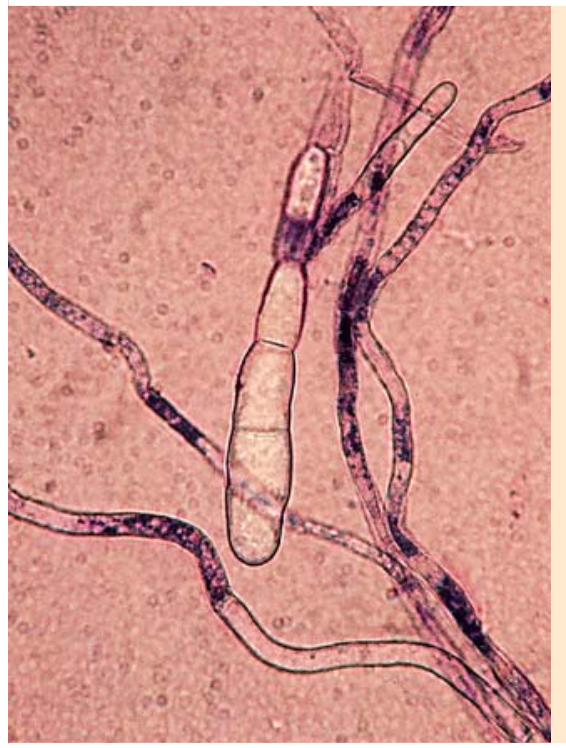

Abb.66 Makroconidie von Epidermophyton floccosum (stark vergrößert).

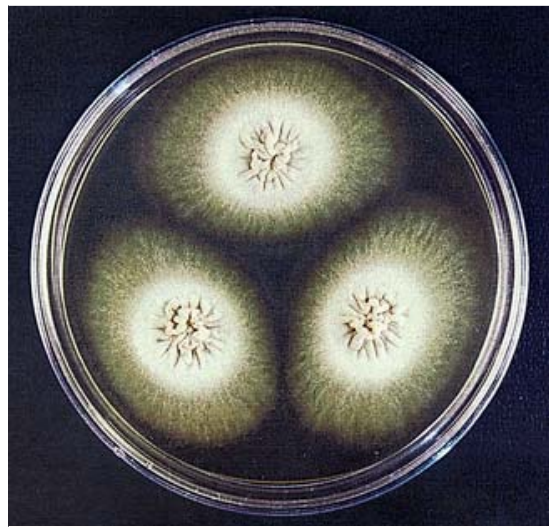

Abb.67 Epidermophyton floccosum auf Sabouraud-MaltoseAgar. 34 Tage alte, cremefarbene, samtige, zentral gefurchte Drillingskultur mit strahlenförmig auslaufenden gelb-grünlichen Hyphenbündeln.

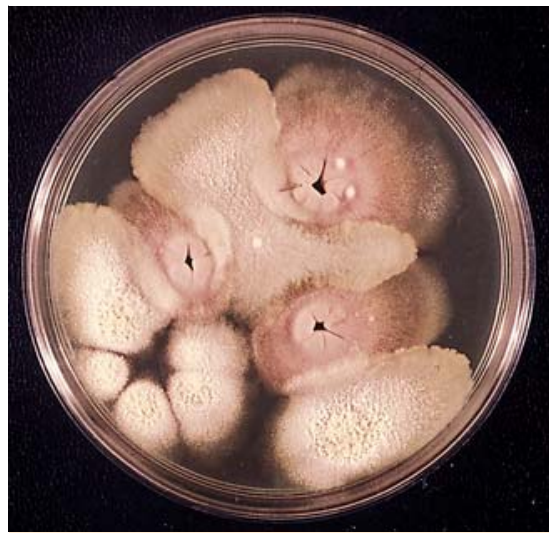

Abb. 68 Epidermophyton floccosum mit kleinen pleomorphen Flaumflöckchen und Scopulariopsis brevicaulis auf SabouraudMaltose-Agar.

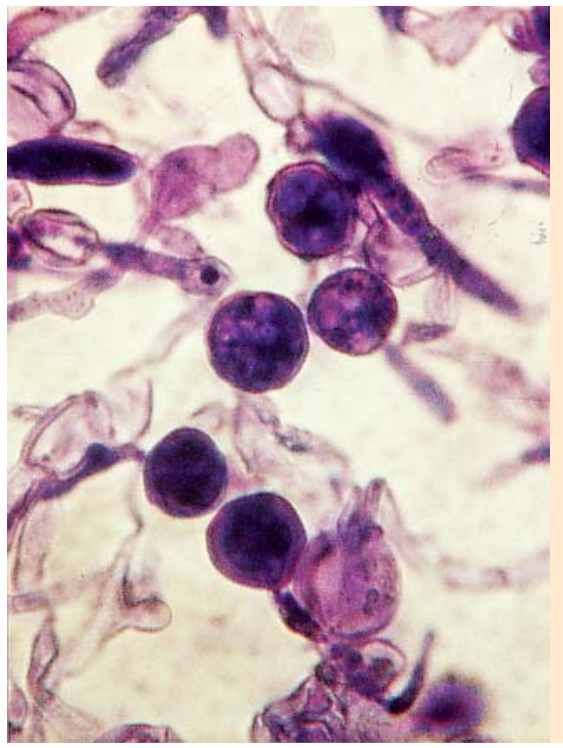

Abb.69 Chlamydosporen von Epidermophyton floccosum (stark vergrößert).

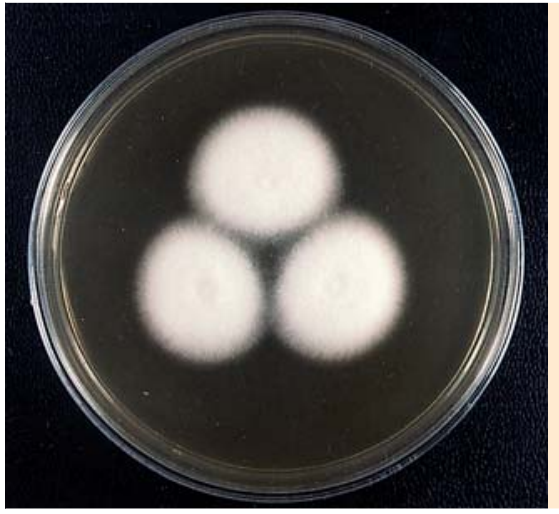

Abb.70 Microsporum audouinii auf KimmigAgar. 9 Tage alte, weiße Drillingskultur mit lockerem, watteähnlichem Luftmyzel.

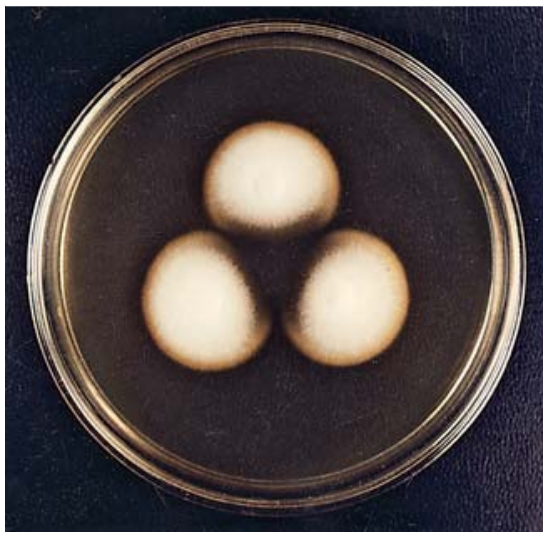

Abb.71 Microsporum

audouinii auf Sabouraud-Maltose-Agar. 12 Tage alte, flache Drillingskultur mit weißer, feinflaumiger Oberfläche und abgesetztem Randsaum. 


\section{Microsporum canis}

Perfektes Stadium: Nannizzia otae

Synonym: Microsporum felineum, Microsporum lanosum, Microsporum obesum

Microsporum canis ist der häufigste Mikrosporie-Erreger. Dieser zoophile Dermatophyt wird meist von infizierten Haustieren (vor allem Katzen) auf den Menschen übertragen und verursacht ein ähnliches Krankheitsbild wie Microsporum audouinii, jedoch mit stärker ausgeprägten Entzündungserscheinungen. Bevorzugte Lokalisationen sind neben dem behaarten Kopf das Gesicht, die Arme, der Hals und die Brust.

Makromorphologie: Schnell wachsende, weiße bis orange-gelbe, flache Kolonien, mit flaumig-wolliger, feinstrahliger, im Zentrum etwas dichterer Oberfläche. Manche Kulturen bilden ein radiärfaltiges Oberflächenprofil ( $\bullet$ Abb.74 und $\odot$ Abb. 75). Die Kulturunterseite ist gelb, gelbbraun oder orange.

Mikromorphologie: Makroconidien sind je nach Stamm in unterschiedlicher Anzahl vorhanden, dick- und rauwandig, spindelförmig und meist 4- bis 9-fach septiert ( $\bullet$ Abb.76 und $\odot$ Abb. 77). Mikroconidien sind selten, birnen- oder keulenförmig und lateral an den Hyphen angeordnet. Terminale und interkalare Chlamydosporen werden gelegentlich in älteren Kulturen gebildet. Woodlicht: grüne Fluoreszenz-Färbung.

\section{Microsporum cookei}

Perfektes Stadium: Nannizzia cajetana

Microsporum cookei ist ein geophiler Dermatophyt, der nur sehr selten als Krankheitserreger in Erscheinung tritt.

Makromorphologie: Schnell wachsende, weißlich-cremefarbene bis hellgelbe, flache Kolonien mit puderig-körniger Oberfläche. Der Kolonienrand ist von sehr feinen, strahlenförmig auslaufenden, oft rötlich pigmentierten Hyphenbündeln umgeben $(\boldsymbol{Q} \mathbf{A b b}$. 78 und $\odot$ Abb. 79). Die Kulturunterseite ist purpurrot.

Mikromorphologie: Microsporum cookei bildet zahlreiche, dickund rauwandige, gedrungen wirkende, spindelförmige, meist 5bis 7-, selten 10-zellige Makroconidien ( $\bullet$ Abb.80).

Mikroconidien sind in unterschiedlicher Anzahl vorhanden, oval bis birnenförmig und in Akladiumform angeordnet.

\section{Microsporum equinum ${ }^{6}$}

Microsporum equinum ist ein bevorzugt in Großbritannien, vor allem bei Pferden vorkommender, zoophiler Dermatophyt.

Makromorphologie: Schnell wachsende, grau-weiße, flache Kolonien, mit kurzflaumiger, feinstrahliger, glatter oder radiär gefurchter Oberfläche $(\bullet \mathbf{A b b} .81$ und $\bullet \mathbf{A b b} . \mathbf{8 2})$. Die Kulturunterseite ist hellgelb.

Mikromorphologie: Mikroskopisch charakteristisch für diese Pilzart sind zahlreiche, dick und rauwandige, spindelförmige, überwiegend 5-zellige Makroconidien ( $\bullet$ Abb. 83).

Mikroconidien sind je nach Stamm in unterschiedlicher Anzahl vorhanden, birnenförmig und zumeist lateral an den Hyphen angeordnet. Woodlicht: grüne Fluoreszenz-Färbung.

\footnotetext{
${ }^{6}$ Microsporum equinum wurde mit Microsporum canis zusammengelegt.
}

\section{Microsporum gypseum}

Perfekte Stadien: Nannizzia gypsea, Nannizzia incurvata Synonym: Achorion gypseum, Microsporum xanthodes

Microsporum gypseum ist ein geophiler Dermatophyt und Erreger der sogenannten „Gärtner-Mikrosporie“.

Makromorphologie: Schnell wachsende, hellgelbe, sandfarbene oder zimtbraune Kolonien, mit flachem, anfangs leicht flaumigem, später jedoch puderig-körnigem bis gipsigem Myzelgeflecht ( $\odot$ Abb.84 und $\odot$ Abb.85). Die Kulturunterseite ist gelblich bis rotbraun ( $\odot \mathbf{A b b} .86)$.

Mikromorphologie: Typisch für diese Pilzart sind sehr viele, relativ breite, dünn- und überwiegend rauwandige, spindelförmige, 4- bis 6-zellige Makroconidien, die oft büschelweise zusammenstehen ( $\bullet$ Abb. 87).

Mikroconidien sind selten, oval bis birnenförmig und lateral an den Hyphen angeordnet.

\section{Microsporum nanum}

Perfektes Stadium: Nannizzia obtusa

Synonym: Microsporum gypseum var. nana

Dieser geophile Dermatophyt wird gelegentlich bei hautkranken Schweinen nachgewiesen. Menschliche Infektionen sind relativ selten und stets auf einen Kontakt mit Tieren zurückzuführen.

Makromorphologie: Schnell wachsende, weiße, manchmal auch hellgelbe bis ockergelbe Kolonien von feinflaumiger bis puderigkörniger Konsistenz ( $\bullet$ Abb. 88). Die Kulturunterseite ist orange bis rotbraun. Der Kulturhabitus ist dem von Microsporum gypseum sehr ähnlich.

Mikromorphologie: Mikroskopisch charakteristisch für diese Pilzart sind zahlreiche, rauwandige, relativ kleine, ovale, 1- bis 3-zellige Makroconidien ( $\bullet$ Abb. 89).

Mikroconidien sind selten, birnen- oder keulenförmig und in Akladiumform angeordnet.

\section{Abstract}

\section{Introduction to the Diagnosis of Human Pathogenic Fungi - Part 2: Identification of Dermatophytes \\ $\checkmark$}

Dermatophytes are keratinophilic fungi that cause mycoses on skin, hair and nails only. They do not normally penetrate nonkeratinised layers.

The morphology of macroconidia is used to distinguish between the three genera Trichophyton, Epidermophyton and Microsporum. Macroconidia are smooth-walled in Trichophyton and Epidermophyton and rough-walled in Microsporum. Trichophyton has cylindrical, Epidermophyton clavate, and Microsporum fusiform macroconidia.

The macroconidia and microconidia as well as a number of other characteristic microstructures of these skin fungi are formed during the imperfect (asexual) stage. In addition, perfect (ascogenous or sexual) fruiting structures have been identified in many dermatophytes. This makes it possible to classify these fungi. Dermatophytes that do not form sexual fruiting bodies or whose fruiting bodies are unknown are classified as „fungi imperfecti“ (deuteromycota). 


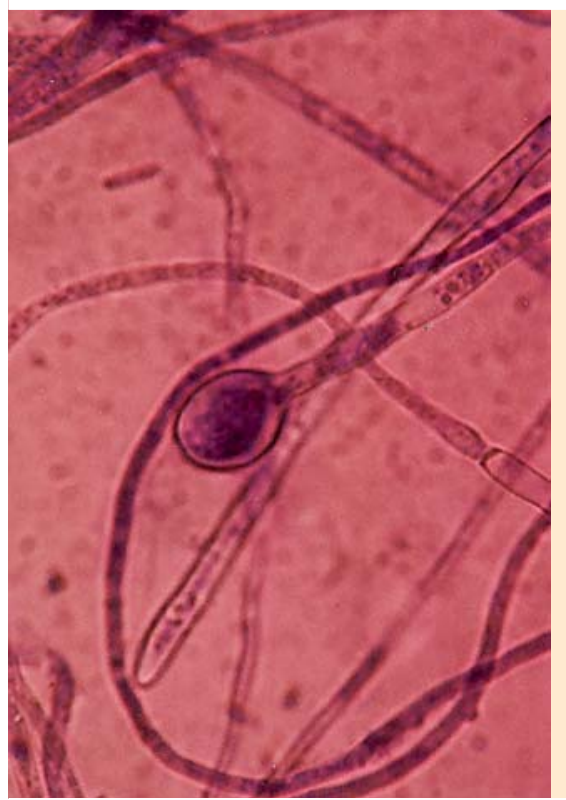

Abb.72 Terminal gebildete Chlamydospore von Microsporum audouinii (stark vergrößert).

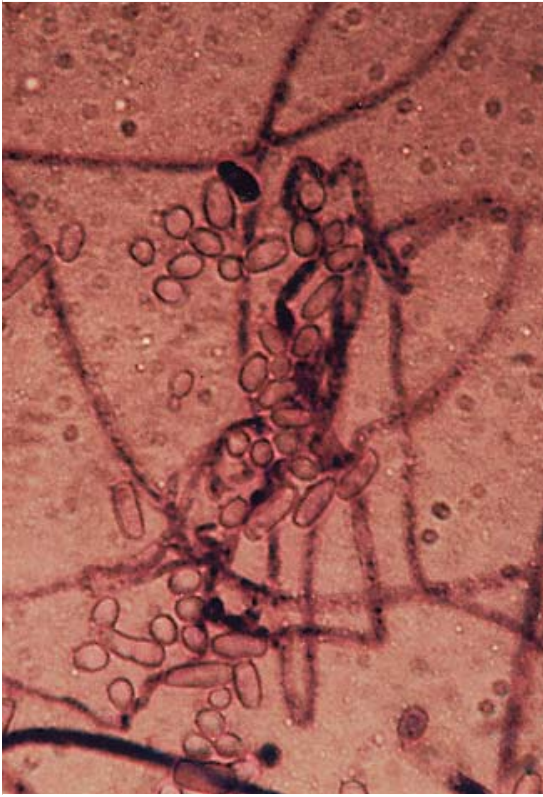

Abb.73 Mikroconidien von Microsporum audouinii (stark vergrößert).

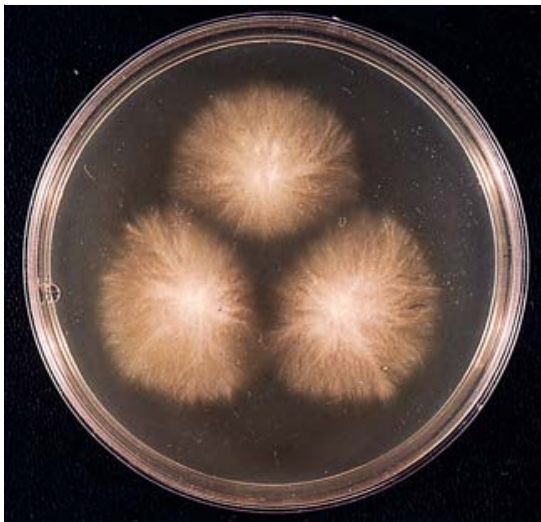

Abb.74 Microsporum canis auf SabouraudMaltose-Agar. 13 Tage alte, flache, gelblichweiße, feinflaumige Drillingskultur mit strahlenförmig auslaufenden Hyphenbündeln.

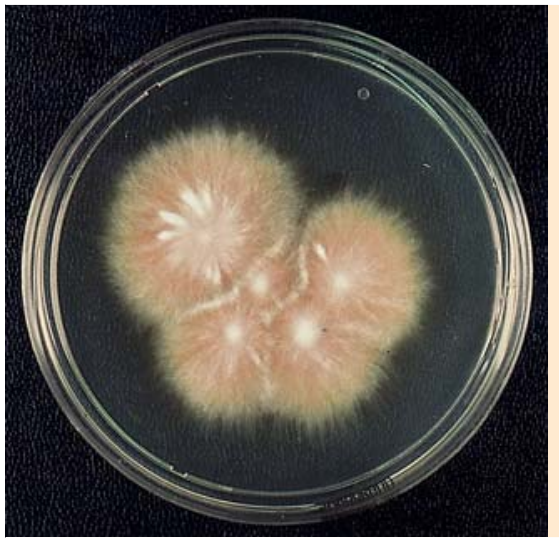

Abb.75 Microsporum canis auf SabouraudGlukose-Agar (2\%). 14 Tage alte, flache, gelblich-weiße Reinkultur mit flaumig-wolliger, feinstrahliger Oberfläche.

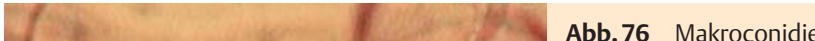
von Microsporum canis (stark vergrößert).

bb.77 Makroconi-

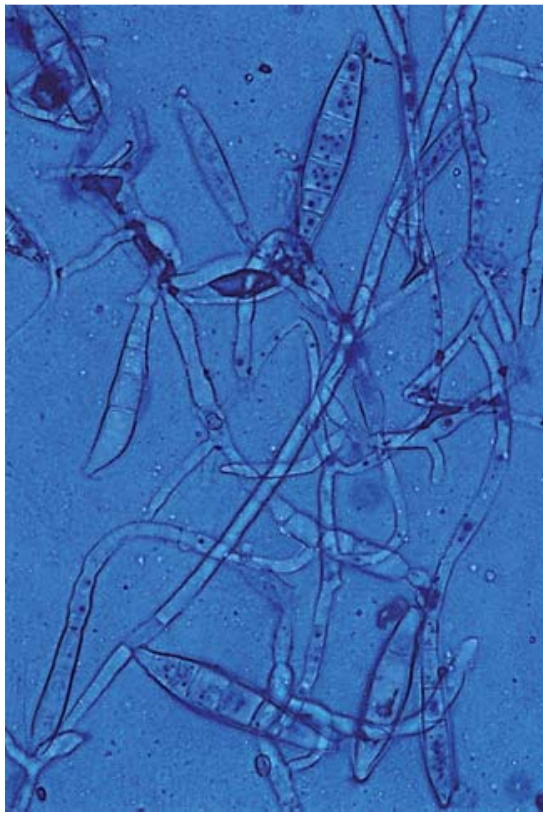
canis. 


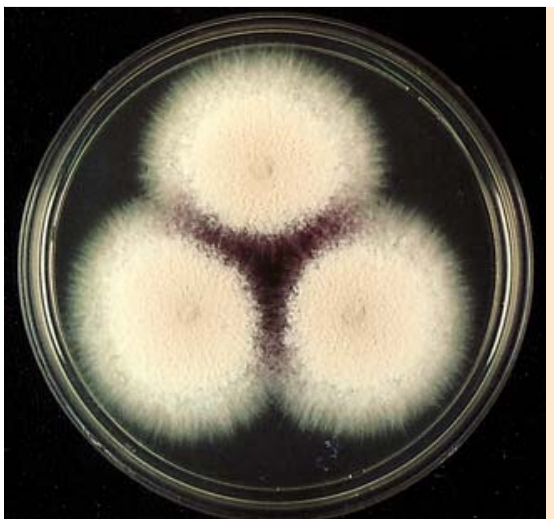

Abb.78 Microsporum cookei auf KimmigAgar. 13 Tage alte, flache, cremefarbene, puderig-körnige Drillingskultur mit flaumigwolliger peripherer Zone.

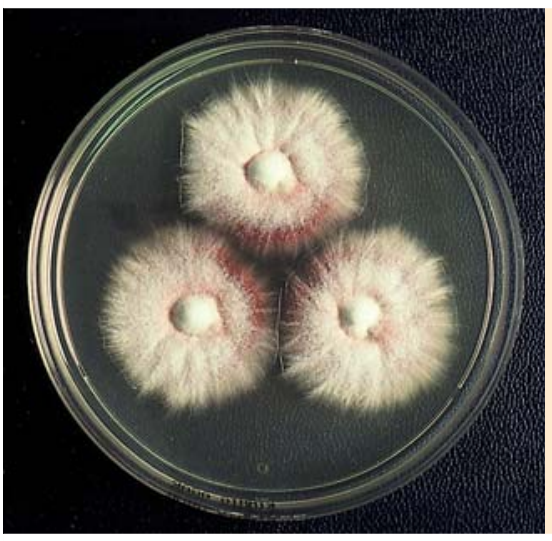

Abb.79 15 Tage alte, cremefarbene, flache, puderig-körnige, im Randbereich stellenweise schwach rötlich pigmentierte Drillingskultur mit strahlenförmig auslaufenden Hyphenbündeln eines anderen Sammlungsstammes auf MycoselAgar.

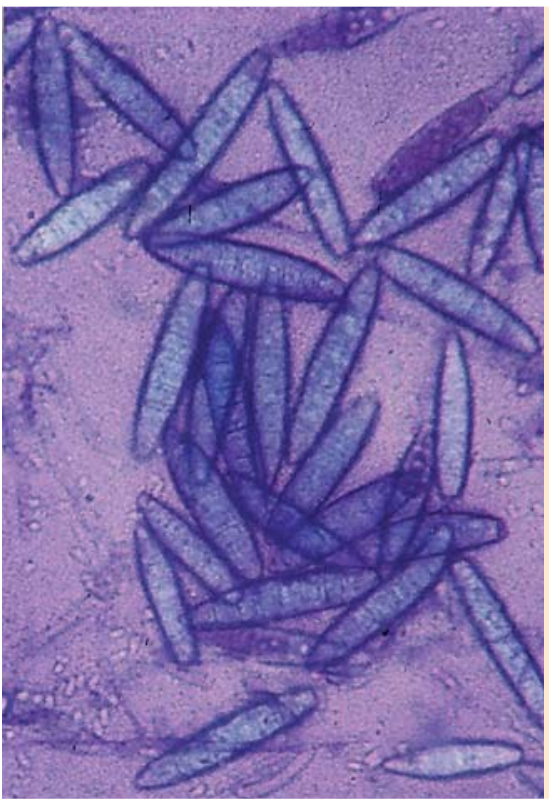

Abb. 80 Makroconidien von Microsporum cookei (stark ver-

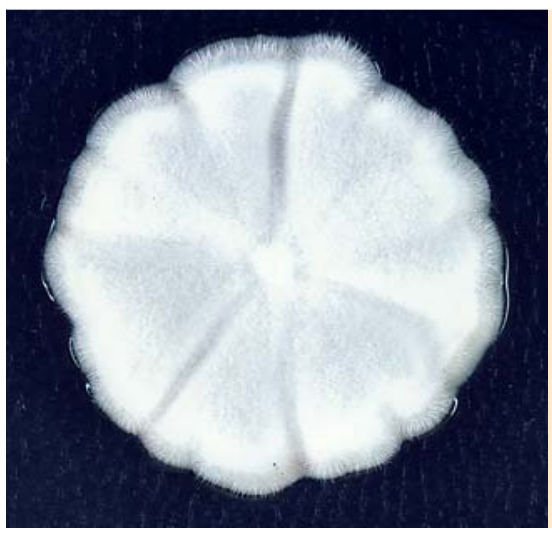

Abb. 81 Microsporum equinum auf Sabouraud-Glukose-Agar (2\%). 14 Tage alte, grauweiße Monokultur mit feinflaumiger, radiär gefurchter Oberfläche. größert).

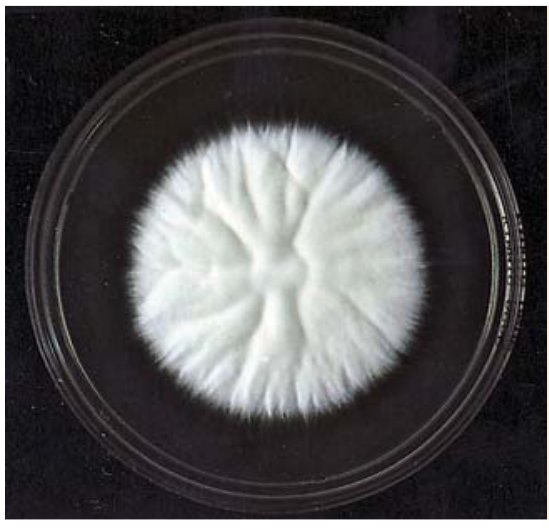

Abb. 82 Microsporum equinum auf KimmigAgar. 15 Tage alte, grau-weiße Monokultur mit samtiger bis kurzflaumiger, unregelmäßig radiär gefurchter Oberfläche.

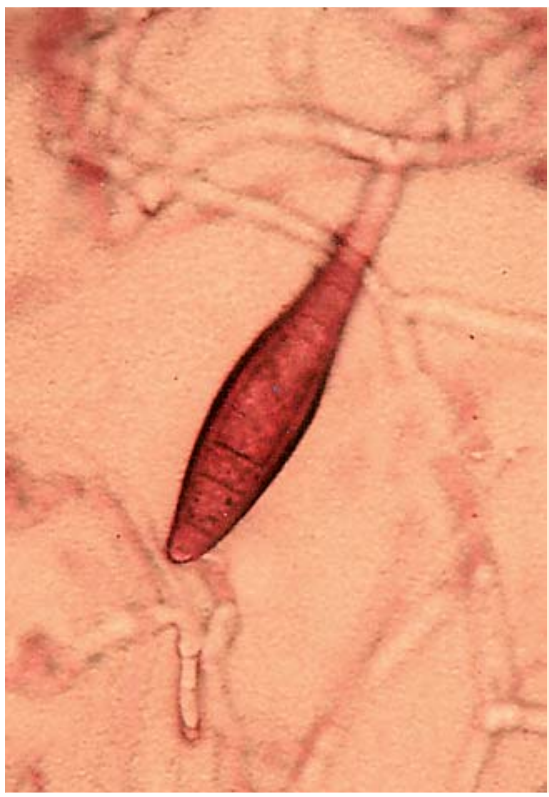

Abb. 83 Makroconidie von Microsporum equinum (stark vergrößert).

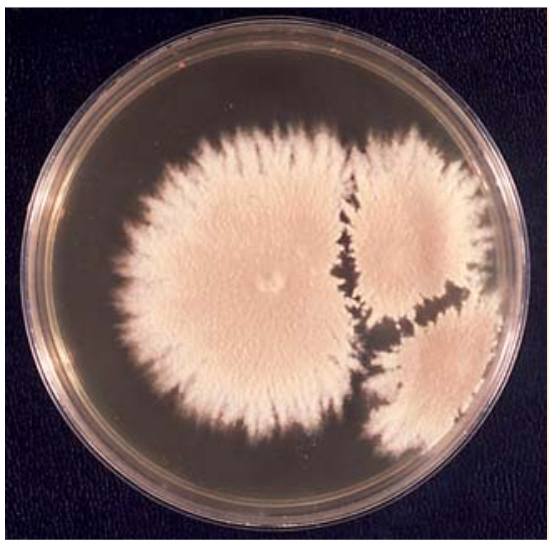

Abb.84 Microsporum gypseum auf KimmigAgar. 12 Tage alte Reinkultur mit feinbis grobkörniger Oberfläche und zerfranstem Rand.

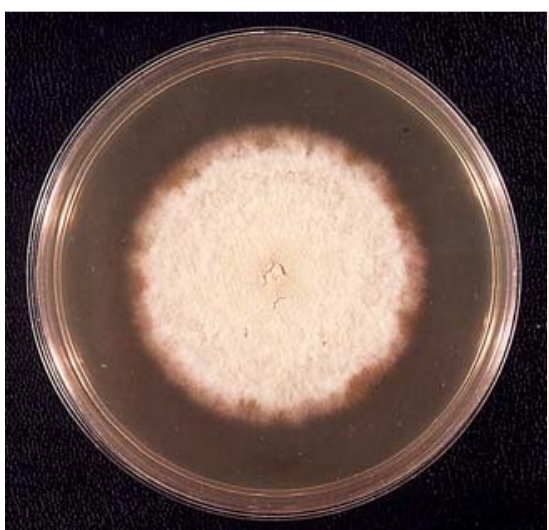

Abb. 8513 Tage alte Monokultur mit samtigpuderiger Oberfläche eines anderen Sammlungsstammes auf Kimmig-Agar. 


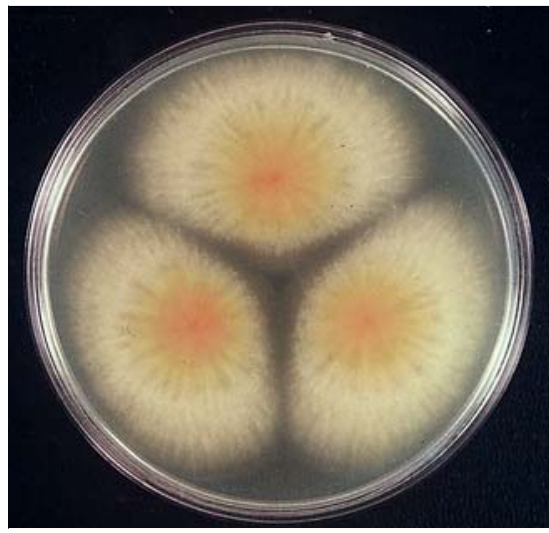

Abb. 86 Rotgelb pigmentierte Kulturunterseite von Microsporum gypseum.

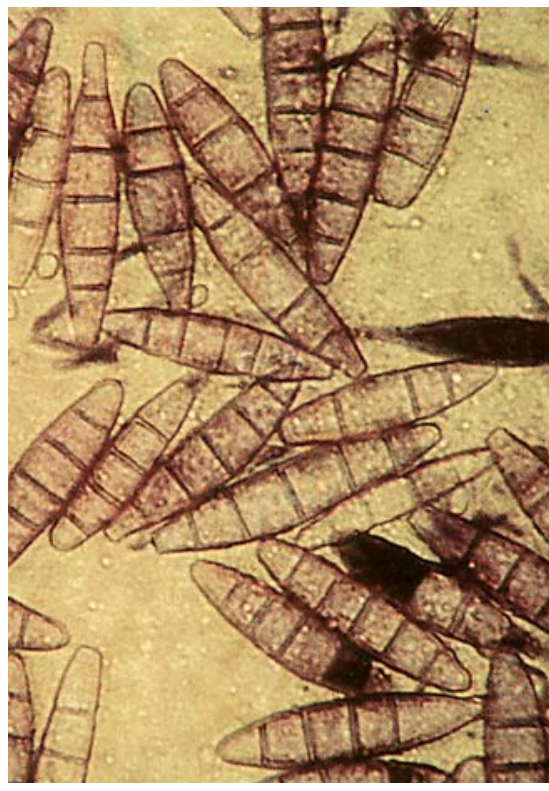

Abb.87 Makroconidien von Microsporum gypseum (stark vergrößert).
Dermatophytes are classified as geophilic dermatophytes, which live primarily in the soil as saprophytes, zoophilic dermatophytes, which are usually found in warm-blooded animals, and anthropophilic dermatophytes, which are well adapted to humans.

Fungal diseases caused by dermatophytes, which are known as dermatophytoses, are - at least in the industrialised world - significantly on the rise and present a particular challenge for physicians and their assistants because successful antifungal therapy necessitates a prior and exact diagnosis.

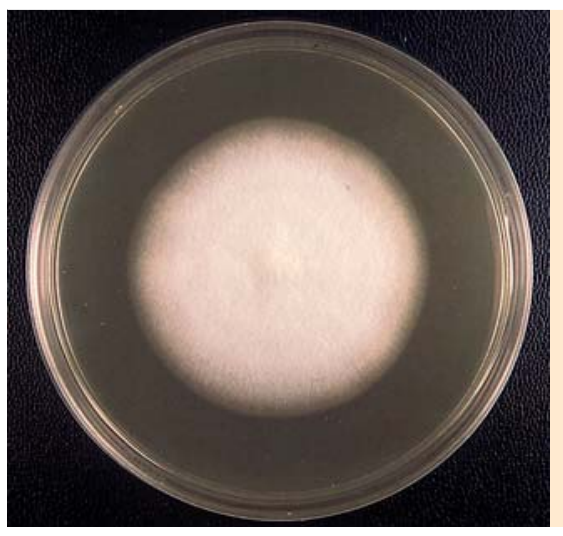

Abb. 88 Microsporum nanum auf KimmigAgar. 22 Tage alte, weiße Monokultur mit puderig-körniger Oberfläche.

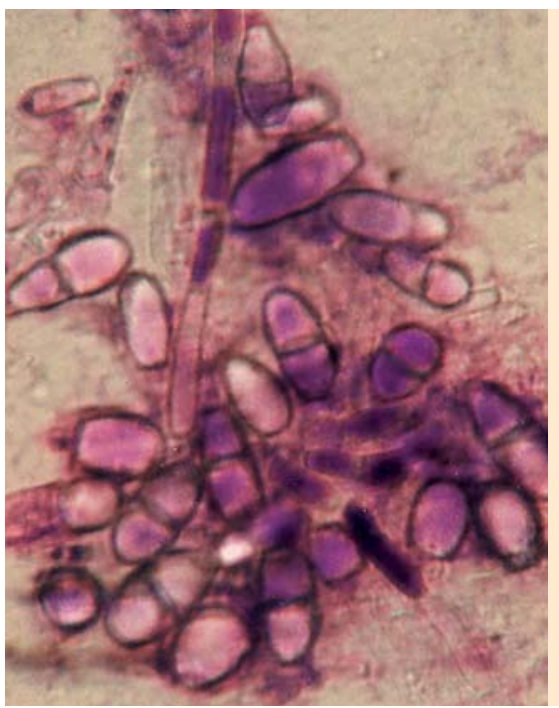

Abb. 89 Makroconidien von Microsporum nanum (stark vergrößert).

\section{Literatur}

1 Seeliger HPR, Heymer T. Diagnostik pathogener Pilze des Menschen und seiner Umwelt. Stuttgart: Thieme; 1981

2 Rohde B, Hartmann G. Mykologie in Beispielen. Hamburg: Scherax-Arzneimittel; 1989

3 Nolting S, Fegeler K. Medizinische Mykologie. 3. Aufl. Heidelberg: Springer; 1987

4 Sorhage B, Ksoll A-M. Pilzerkrankungen durch Trichophyton tonsurans. Wehrmedizinische Monatsschrift 2003; 5: 103-107

5 Tietz H-J, Ulbricht H. Humanpathogene Pilze der Haut und Schleimhäute. Hannover: Schlütersche; 1999

6 Ksoll A-M, Sorhage B. Ein interessanter Dermatophyt: Trichophyton tonsurans als Erreger der „Tinea gladiatorum“. MTA Dialog 2004; 10 : $762-765$

7 Darai G, Handermann M, Sonntag HG. Lexikon der Infektionskrankheiten des Menschen. Heidelberg: Springer; 2009

8 Frey $D$, Oldfield $R$, Bridger $R$. Farbatlas pathogener Pilze. Hannover: Schlütersche; 1985

9 Heber W, Hauss H. Mykologische Techniken in der ärztlichen Praxis. München: Schwarzeck; 1983 